\title{
$W$-operator learning using linear models for both gray-level and binary inputs
}

\author{
Igor dos Santos Montagner
}

TESE APRESENTADA

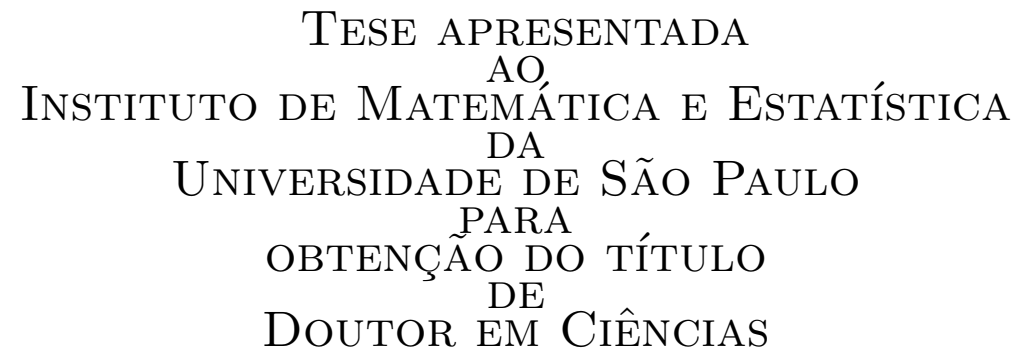

Programa: Ciência da Computação

Orientador: Prof. Dr. Roberto Hirata Jr.

Co-orientadora: Prof. Dra. Nina S. T. Hirata

Durante o desenvolvimento deste trabalho o autor recebeu auxílio da FAPESP, projetos 2011/23310-0 e 2014/21692-0.

São Paulo, February 2017 


\section{$W$-operator learning using linear models for both gray-level and binary inputs}

Esta versão da dissertação/tese contém as correções e alterações sugeridas pela Comissão Julgadora durante a defesa da versão original do trabalho, realizada em 12/06/2017. Uma cópia da versão original está disponível no Instituto de Matemática e Estatística da Universidade de São Paulo.

Comissão Julgadora:

- Prof. Dr. Roberto Hirata Jr. (orientador) - IME-USP

- Prof. Dr. Roberto Cesar Jr. - IME-USP

- Prof. Dr. Stéphane Canu - INSA-Rouen

- Prof. Dr. Alexandre Falcão - UNICAMP

- Prof. Dr. André C. de Carvalho - ICMC-USP 


\section{Abstract}

MONTAGNER, Igor S., $W$-operator learning using linear models for both gray-level and binary inputs, 2017. Thesis (Phd) - Instituto de Matemática e Estatística, Universidade de São Paulo

Image Processing techniques can be used to solve a broad range of problems, such as medical imaging, document processing and object segmentation. Image operators are usually built by combining basic image operators and tuning their parameters. This requires both experience in Image Processing and trial-and-error to get the best combination of parameters.

An alternative approach to design image operators is to estimate them from pairs of training images containing examples of the expected input and their processed versions. By restricting the learned operators to those that are translation invariant and locally defined ( $W$-operators) we can apply Machine Learning techniques to estimate image transformations. The shape that defines which neighbors are used is called a window.

$W$-operators trained with large windows usually overfit due to the lack sufficient of training data. This issue is even more present when training operators with gray-level inputs. Although approaches such as the twolevel design, which combines multiple operators trained on smaller windows, partly mitigates these problems, they also require more complicated parameter determination to achieve good results.

In this work we present techniques that increase the window sizes we can use and decrease the number of manually defined parameters in $W$ operator learning. The first one, KA, is based on Support Vector Machines and employs kernel approximations to estimate image transformations. We also present adequate kernels for processing binary and gray-level images. The second technique, NILC, automatically finds small subsets of operators that can be successfully combined using the two-level approach.

Both methods achieve competitive results with methods from the literature in two different application domains. The first one is a binary document processing problem common in Optical Music Recognition, while the second is a segmentation problem in gray-level images. The same techniques were applied without modification in both domains.

Keywords: $W$-operator learning; Support Vector Machines; Linear classification methods; Machine Learning; Image Processing 


\section{Resumo}

MONTAGnER, Igor S., Aprendizado de $W$-operadores usando modelos lineares para imagens binárias e em níveis de cinza. 2017. Thesis (Phd) - Instituto de Matemática e Estatística, Universidade de São Paulo

Processamento de imagens pode ser usado para resolver problemas em diversas áreas, como imagens médicas, processamento de documentos e segmentação de objetos. Operadores de imagens normalmente são construídos combinando diversos operadores elementares e ajustando seus parâmetros. Uma abordagem alternativa é a estimação de operadores de imagens a partir de pares de exemplos contendo uma imagem de entrada e o resultado esperado. Restringindo os operadores considerados para o que são invariantes à translação e localmente definidos ( $W$-operadores), podemos aplicar técnicas de Aprendizagem de Máquina para estimá-los. O formato que define quais vizinhos são usadas é chamado de janela.

$W$-operadores treinados com janelas grandes frequentemente tem problemas de generalização, pois necessitam de grandes conjuntos de treinamento. Este problema é ainda mais grave ao treinar operadores em níveis de cinza. Apesar de técnicas como o projeto dois níveis, que combina a saída de diversos operadores treinados com janelas menores, mitigar em parte estes problemas, uma determinação de parâmetros complexa é necessária.

Neste trabalho apresentamos duas técnicas que permitem o treinamento de operadores usando janelas grandes. A primeira, KA, é baseada em Máquinas de Suporte Vetorial (SVM) e utiliza técnicas de aproximação de kernels para realizar o treinamento de $W$-operadores. Uma escolha adequada de kernels permite o treinamento de operadores níveis de cinza e binários. A segunda técnica, NILC, permite a criação automática de combinações de operadores de imagens. Este método utiliza uma técnica de otimização específica para casos em que o número de características é muito grande.

Ambos métodos obtiveram resultados competitivos com algoritmos da literatura em dois domínio de aplicação diferentes. O primeiro, Staff Removal, é um processamento de documentos binários frequente em sistemas de reconhecimento ótico de partituras. O segundo é um problema de segmentação de vasos sanguíneos em imagens em níveis de cinza.

Palavras chave: Projeto automático de $W$-operadores; Máquinas de Suporte Vetorial; Aprendizado de Máquina; Processamento de imagens. 


\section{Acknowledgments}

First and foremost, I would like to thank Roberto Hirata Jr. for his support and friendship during the last 6 years. Besides being a great advisor, he has opened many doors for me and presented me with opportunities I never expected to have. I will be forever grateful for his dedication and patience.

I would also like to thank Nina Hirata and Stéphane Canu for their involvement in this work. Nina gave me much more time and attention than I could ever expect from a co-advisor. In fact, I sometimes suspect that I give her more work than her own students. Nonetheless she always responded with patience and encouragement. To Stéphane I express my deepest thanks for the opportunity to work with him in France. The time I spent at INSA-Rouen is very dear to me and I grew a lot both professionally and personally. Both Stéphane and Nina put their hearts into whatever they did, including this collaboration. It was a pleasure to work with them.

Many friends have given me the pleasure of their company during this journey. Andrew Kurauchi, Gustavo Vilela, Vinicius Daros, Henrique Stagni and many others not cited here by name have made my life easier just by being present and listening to me.

My lab colleagues, including Fernando Andrade, Caio Braz and Lucy Choque, also provided me many laughters and relaxing moments. It was a pleasure to work in your company.

Besides Roberto and Nina, my wife Karina was the only other person to see the development of this work from its very beginning to its end. Although I can not say it was a rough ride, it certainly was not smooth. She supported me and endured my bad mood during hard times. She also motivated me with a unwavering confidence that I could do whatever I set my mind to.

I also thank the support of my parents. They have provided me with stability to focus on this work even though they do not have the slightest clue about what I do. They have taught me and my sister to value persistence over talent and that hard work is necessary to achieve what is important to us.

I thank FAPESP for supporting this work (grant numbers 2011/23310-0 e 2014/21692-0). I have received many good opportunities thanks to the two research grants I received, including spending an year abroad in France. 
Last, I would like to thank God for putting all these wonderful people in my life. I have received much more from these people than I could ever hope to give them back and I pray that one day I will be able to pay it forward to many others. 


\section{Contents}

List of Figures ix

List of Tables $\quad$ xi

List of Symbols $\quad$ xiii

\begin{tabular}{lll}
\hline & Introduction & 1
\end{tabular}

1.1 Objectives and proposed methods. . . . . . . . . . . . . 4

1.2 Resulting publications $\ldots \ldots \ldots \ldots \ldots$

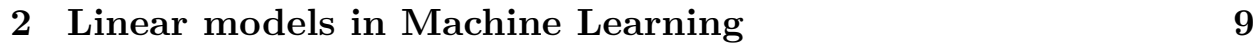

2.1 Logistic Regression . . . . . . . . . . . . . . . . . . . . . . . . 11

2.2 Support Vector Machines $\ldots \ldots \ldots \ldots$

2.3 Nonlinear decisions using feature maps . . . . . . . . . . . . 16

\begin{tabular}{|lll}
\hline 3 & Fundamentals of W-operator learning & 17
\end{tabular}

3.1 Learning formulation . . . . . . . . . . . . . . . . 18

3.2 Two level operators . . . . . . . . . . . . . . . . . . . 21

4 SVMs applied to $W$-operator learning 23

4.1 Kernel Support Vector Machines . . . . . . . . . . . . . . . . 23

4.2 Approximating a kernel's implicit feature map. . . . . . . . . 25

4.3 Kernels applied to image data . . . . . . . . . . . . . . 28

\begin{tabular}{|lll}
5 & Two-level operator optimization & 31
\end{tabular}

5.1 Previous approaches . . . . . . . . . . . . . . . 32

5.2 Two-level design based on window ranking . . . . . . . . . . . 35

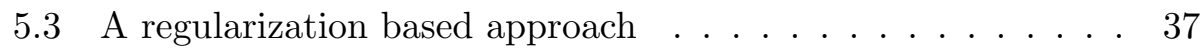

6 Experiments 43

6.1 Kernel hyperparameter determination for Nyström approximations in KA . . . . . . . . . . . . . . 44

6.2 Random behavior of the KA algorithm . . . . . . . . . . . 44

$6.3 \quad$ Determining $\lambda$ for NILC $\ldots \ldots \ldots \ldots$. . . . . . . . . . . . 48 
6.4 Generalization performance $\ldots \ldots \ldots \ldots$

$\begin{array}{lll}7 & \text { Applications } & 53\end{array}$

7.1 Staff removal . . . . . . . . . . . . . . . . . 53

7.2 Blood vessel segmentation in retinal images . . . . . . . . . 59

$\begin{array}{lll}8 & \text { Conclusion } & 63\end{array}$

\begin{tabular}{ll}
\hline Bibliography & 67
\end{tabular} 


\section{List of Figures}

1.1 Example of input-output image pair. . . . . . . . . . . . 2

1.2 Visually similar images have different pixel representations. . 4

2.1 Trade-off between approximation and generalization. . . . . . 11

2.2 The optimal separating hyperplane $w$ is the one that maximizes the margin $C$ between the hyperplane and the closest training instances. . . . . . . . . . . . . . 13

3.1 Summary of the learning process for $W$-operators. $\ldots \ldots$. . . 19

\begin{tabular}{lll}
\hline 3.2 & Illustration of the learning process for second-level operators. 22
\end{tabular}

4.1 Products of binary features detect patterns in image data. . . 29

4.2 Examples of gray-level image patches extracted. . . . . . . . . 30

5.1 Windows used in WER. Black points are used as features in

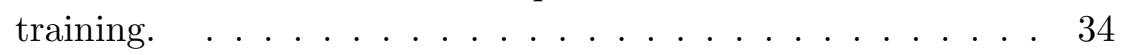

6.1 Validation performance for different hyperparameters (KA). . $\quad 45$

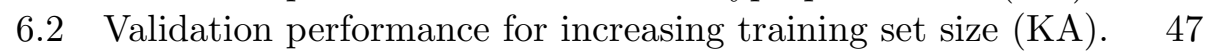

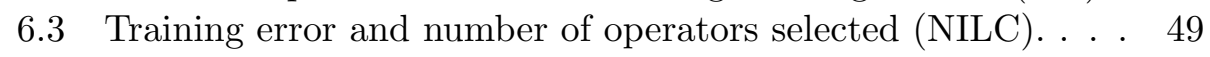

6.4 Training error and number of operators selected (NILC + KA). 50

7.1 Input-output pairs for the staff removal problem. . . . . . . . 53

7.2 Reference windows for staff removal. Black points are used as features in training. . . . . . . . . . . . 54

7.3 Point frequency for a set of Relief rankings. . . . . . . . . . . 56

7.4 Windows found by the FS method. Black points are used as features in training. . . . . . . . . . . . . . 56

7.5 Example of the output of different methods in the staffs dataset. 58

7.6 Input-output pair for the vessel segmentation task. . . . . . . 60

7.7 Windows selected by NILC-DT. There is not a clear pattern in the pixel distribution. Black points are used as features in

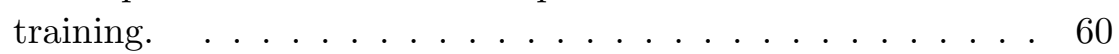

7.8 Result images for the DRIVE dataset. . . . . . . . . . . 62 


\section{List of Tables}

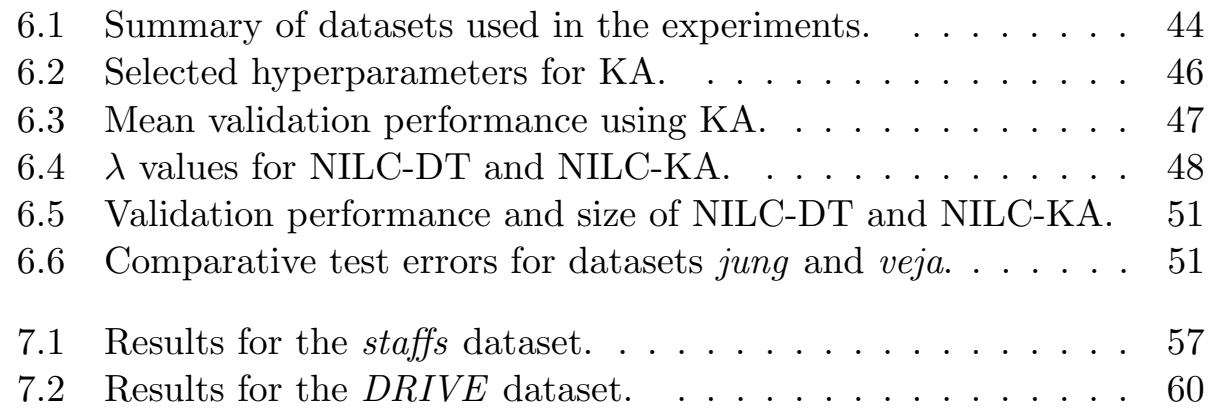




\section{List of Symbols}

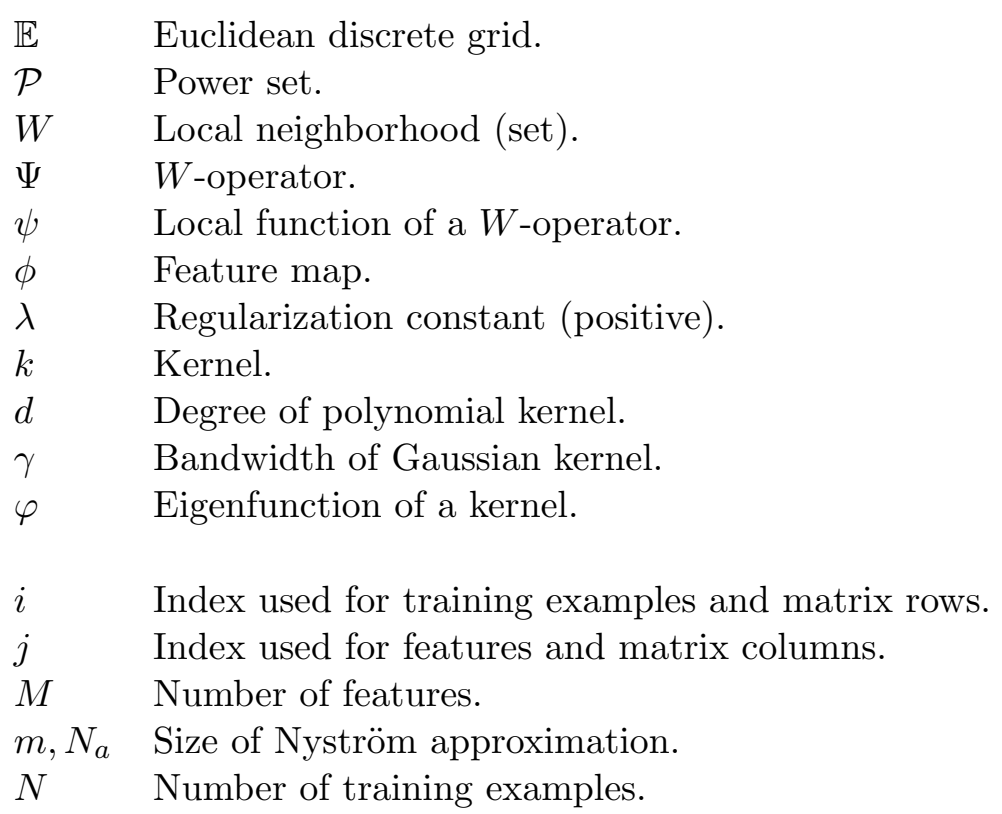




\section{Chapter 1}

\section{Introduction}

In the last decade there has been an explosion of image data available. However, our capacity to extract useful information from these images is, by far, much smaller than our capacity to store them. Most of the information that these images carry stays hidden in these huge datasets. For this reason, the extraction of high-level information from images has been extensively studied in the last decade for many different applications, including medical imaging [SAN ${ }^{+}$04], underwater object detection [HFL16] and document analysis [CCZTR16].

Image Processing studies lower level transformations between images and can be seen as a necessary step in knowledge extraction pipelines. These image transformations, more frequently called image operators, usually employ methods that exploit characteristics intrinsic to a task or domain of application. Thus, designing image operators also requires some knowledge of the application itself. Besides that, images from different domains may have very different characteristics, resulting in little intersection between methods devised for different domains. For instance, techniques used to process medical images are hardly useful for document images.

Image operators are also hard to design. Usually one combines different basic operators and adjusts their parameters to obtain the best result possible. For instance, morphological operators analyze images based on a structuring element, which is a shape of interest that may be present in the images. Complex operators can be designed by combining simple operators and carefully chosen structuring elements Soi03. This process is based on trial and error and offers no guarantee on the quality of the resulting operator. If an approach does not work it might be necessary to start from scratch using a different method to improve results. Also, having more images available does not necessarily help, since the process of building the operator is mostly heuristic.

There has been much progress in an alternative way of designing image operators in the last two decades. Instead of using heuristics and previous 


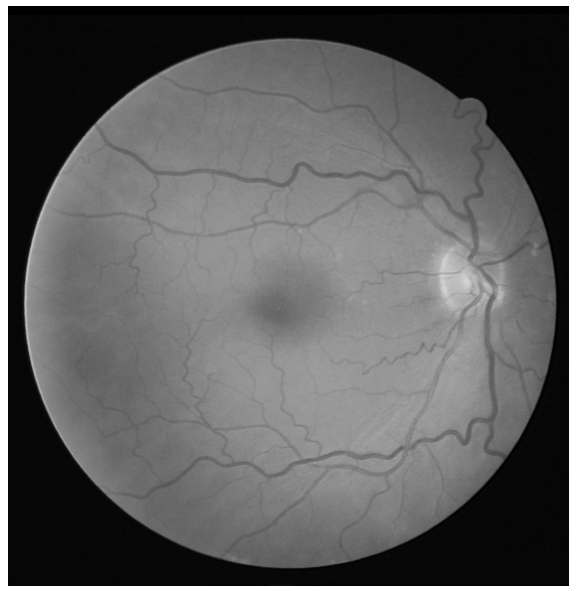

(a) Input

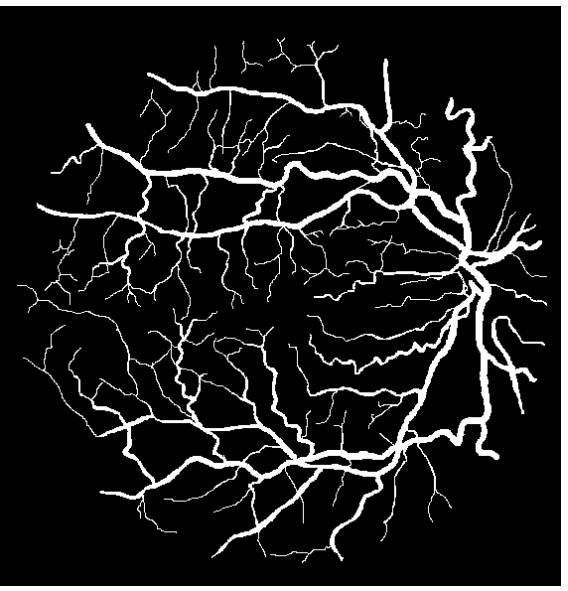

(b) Expected output

Figure 1.1: Input-output pair for the blood vessel segmentation task (from $\left[\mathrm{SAN}^{+} 04\right]$ ).

knowledge, in this approach image operators are learned from pairs of images represeting an image processing task. The first image of the pair is an example a typical input, while the second image is the desired output of the image operator. An example is shown in Figure 1.1, where the task of interest is the segmentation of blood vessels in retinal images. Defining a task in this manner does not impose any limitation on which techniques are used to build an image operator that solves it. In fact, this representation is useful even when designing image operators in the traditional manner, as it provides a ground truth for the designed operator.

Many works have studied the estimation of local image transformations, called $W$-operators, from pairs of input-output images. A $W$-operator is characterized by a local function $\psi$ that classifies image patches extracted from the input image into two or more gray-levels. The shape and size of the extracted image patches is defined by a neighborhood $W$, called window in this context. To produce an output image the local function $\psi$ is applied to patches centered in every pixel in the input image.

Given a set of input-output pairs $\left\{\left(I_{i}, O_{i}\right)\right\}$ (like the ones in Figure 1.1) and a window $W$, Dougherty [Dou92 proposed a method to design $W$ operators that minimizes the squared error between the expected output and the transformed images. In this formulation a $W$-operator is represented via a decomposition using morphological operators. The estimated operator $\psi$ is called the optimal $W$-operator with regard to training set $\left\{\left(I_{i}, O_{i}\right)\right\}$ and window $W$. Many subsequent works [BTHH00, MCB06, HBBD06, Hir09, dSdS10, DH15, have focused on this morphological approach for $W$-operator learning.

The main hyperparameter used by this approach is the window $W$. The 
shape and size of $W$ define what patterns can be observed in the extracted image patches. A small window (i.e. one with a small number of neighboring points) is unlikely to contain sufficient information to detect complex patterns. On the other hand, training using large windows usually results in operators that achieve perfect results in the training set but do not perform well on unseen images. Therefore, learning $W$-operators is a trade-off between window size and generalization performance.

A recent approach for learning operators over large windows is the two-level design [Hir09]. This method combines the output of several $W$ operators into a second level pattern and trains a combination operator to determine the final output. The decision of the second level operator depends, indirectly, on the neighborhood defined by the union of all combined windows. Although this achieves better generalization performance, it also requires much more complicated parameter determination. It is necessary to design windows for several first level operators and take into account how these windows interact when combined. A common heuristic used in many works Hir09, MHH14a, DH15 is to use basic shapes, such as lines and rectangles, as first level windows.

Many works [SHH10, DH15, MHH16] have used feature selection techniques to determine windows for two-level operators. Feature selection can be used to determine first-level windows inside a large domain window $D$, as in [SHH10, MHH16], or to select which operators to combine from a set of candidates [DH15, MHH16]. In general, window determination and classifier training are regarded as separate tasks. Although the combination operator is optimal with regard to the second level patterns, there is no established definition of the optimal two-level operator as a whole with regards to domain window $D$.

Training $W$-operators with gray-level input images poses additional challenges due to an exponential increase of the feature space size. When dealing with binary inputs, the space of all patterns has size $2^{M}$ for windows of size $M$ but it has size $\kappa^{M}$ when considering $\kappa$ gray-levels. Also, visually identical patterns may in fact have slightly different pixel values. The two images in Figure 1.2 are examples of this problem. Even though they are visually similar most of their pixel values differ. The image on the right was obtained by adding noise to the original one (on the left). These issues lead to severe overfitting when training gray-level operators.

To mitigate the increase in feature space size some techniques were developed. Aperture operators [HBBD06] compute representations that are invariant to global brightness changes and resistant to salt and pepper noise. This greatly reduces pattern variations, but it still fails to account for very similar patterns like those in Figure 1.2. Multi-resolution operators [Bru02] analyze the image in many different resolutions to exploit the possibility that many visually similar patterns might have the same representation in coarser scales. However, this method might also discard important information for 


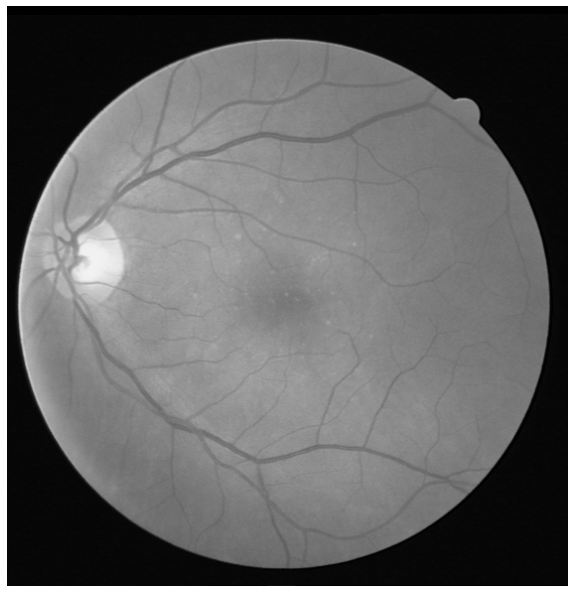

(a) Original

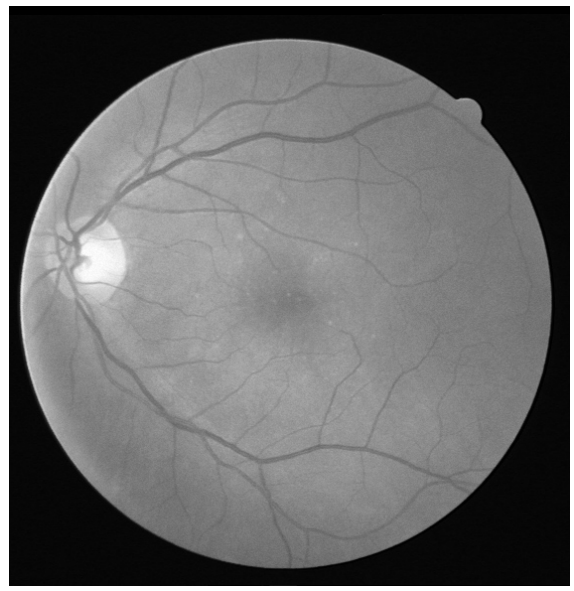

(b) Additive noise

Figure 1.2: Adding noise to gray-level images does not produce significant visual changes (original from $\left[\mathrm{SAN}^{+} 04\right]$ ).

classification. Thus, neither fully addresses the issue. These techniques were most successful on simple tasks such as noise filtering and deblurring, although more complicated experiments were done in very limited settings.

The current state-of-the-art suffers from three main limitations: (i) training operators with gray-level inputs; (ii) training operators defined by large windows and (iii) parameter determination. Many previous methods listed above offer only partial solutions. There is still much room for improvement in all areas.

\subsection{Objectives and proposed methods}

In a more abstract level, $W$-operator learning should not require much intervention from the user and, essentially, "just work". Considerations as whether the input images are gray-level, the size of the window and hyperparameter determination should not prevent the user from obtaining good results using $W$-operator learning. It may be possible to improve results by optimizing these, but a reasonable result should be possible nonetheless. In this thesis we synthesize these statements into four concrete objectives. Fulfilling these objective would bring $W$-operator learning closer to "just works" status.

1. Improve training with gray-level input images and large windows;

2. Reduce parameter determination in two-level operator learning;

3. Create an easy to use library with state-of-the-art methods for $W$ operator learning; 
4. Apply $W$-operator learning to solve research problems with public datasets.

We propose the KA method to improve training with large windows and gray-level input images. KA employs Kernel Support Vector Machines Vap13 to train $W$-operators. Kernel SVMs have strong generalization properties due to the maximum margin property and compute solutions based on the similarity between training patterns. They are also flexible: different kernels produce different similarity measures. To decrease the computational cost of classification and training of Kernel SVMs KA uses the Nyström method [WS01 to compute a feature map $\phi$ that approximates the kernel's implicit feature map. By solving a linear SVM in the space transformed by $\phi$ we approximate the solution of the full kernel SVM problem.

The kernel used in KA defines the similarity measure used in the kernel SVM. For binary inputs a polynomial kernel induces a feature map that resembles a boolean function in the Sum of Products form. Each component of the feature map acts as a boolean pattern matcher. For gray-level images a Gaussian kernel is used to compute a soft threshold between two image patches. If only a few pixels are different the similarity will be closer to 1 . As difference increases similarity decreases sharply. The bandwidth allows to "configure" how much similarity is accepted. Thus, both kernel choices also have interesting visual interpretations.

To decrease parameter determination for two-level operators we have developed NILC, a method that does operator selection and error minimization at the same time. Only two parameters are required: a large window $D$ (called domain window) and a constant $\lambda$ that is related to the number of operators combined. A solution is found by solving a strongly regularized optimization problem. This strategy has been successfully applied in many other ML models, such as LASSO, L1-SVM [HTF09, Elastic-Nets ZH05. NILC uses an optimization algorithm based on a method described in [FYR11 to efficiently consider all possible subwindows of $D$. At each iteration NILC adds and/or removes operators from the combination in order to decrease classification error.

The largest difference between KA and NILC from previous methods is the use of traditional Machine Learning and continuous optimization methods. Previous methods focused on maintaining a morphological representation of the operators, which means representing patterns and image operators as elements in a complete lattice. This led to methods that had interesting visual interpretations. Both KA and NILC also have interpretations as image operations, but do not restrict themselves to discrete representations for patterns and model parameters.

KA and NILC were applied to two different tasks: Staff Removal and Vessel Segmentation. The objective of staff removal is to segment the 
stafflines from the symbols in music scores. We used a large dataset of 4000 images [VKFJ13] to compare NILC and KA with other methods from the literature. The objective of vessel segmentation is to segment vessels from retinal images. We compare our results with other methods from the literature on the DRIVE dataset $\left.\mathrm{SAN}^{+} 04\right]$.

We have explored the staff removal problem in more depth MHH16] and developed the FS method. FS is based on feature selection techniques and is able to identify visual structures present in the music scores. It also obtained state-of-the-art performance when compared to existing methods from the literature.

Finally, we developed and published an open source library called TRIOSlib tri16] containing all methods described in this thesis. It can be easily installed and extended with new methods for $W$-operator learning.

This text is organized as follows. In chapters 2 and 3 we review fundamental aspects of Machine Learning and $W$-operator learning that are relevant to this work. In Chapter 4 we present a method to train operators on large windows using SVMs and kernel approximations. In Chapter 5 we review previous methods for two-level optimization and present NILC, an algorithm for two-level design that does window determination and error minimization at the same time. We explore parameter determination for both proposed methods in Chapter 6. We describe two application domains in Chapter 7 and compare the results achieved by KA and NILC to classic methods from the literature. Finally, the conclusions of our work and future work are presented in Chapter 8.

\section{$1.2 \quad$ Resulting publications}

The methods described in this thesis were published in several conferences and a journal article. We list below the bibliographic details of all our articles.

Journal papers:

1. Montagner, I.S., Hirata, N.S.T.; Hirata Jr, R.; "Staff removal using image operator learning", Pattern Recognition, Volume 63, March 2017, Pages 310-320;

Conference papers:

1. Hirata, N.S.T; Montagner, I.S.; Hirata Jr, R.; "Comics image processing: learning to segment text", First International Workshop on coMics ANalysis, Processing and Understanding Proceedings (2016), part of 23rd International Conference on Pattern Recognition (ICPR), 2016 ; 
2. Montagner, I. S.; Canu, S.; Hirata, N.S.T.; Hirata Jr., R.; "Kernel approximations for $W$-operator learning", Conference on Graphics, Patterns and Images (SIBGRAPI), 2016;

3. Montagner, I. S.; Hirata, N.S.T.; Hirata Jr., R.; Canu, S.; "NILC: a two level learning algorithm with operator selection", IEEE International Conference on Image Processing (ICIP), 2016;

4. Montagner, I. S.; Hirata, R.; Hirata, N.S.T., "Learning to remove staff lines from music score images," IEEE International Conference on Image Processing (ICIP), 2014;

5. Montagner, I. S.; Hirata, R.; Hirata, N.S.T., "A Machine Learning Based Method for Staff Removal," 22nd International Conference on Pattern Recognition (ICPR), 2014;

6. Montagner, I. S.; Hirata, R.; Hirata, N.S.T., "TRIOS - an open source toolbox for training image operators from samples". Workshop of Works in Progress (WIP) in SIBGRAPI 2012 (XXV Conference on Graphics, Patterns and Images);

Tutorials:

1. Montagner, I. S.; Hirata, N.S.T.; Hirata Jr., R.; "Image operator learning and applications", Tutorials of the Conference on Graphics, Patterns and Images (SIBGRAPI-T), 2016;

We have also developed TRIOSlib, an open source research library containing implementations for standard $W$-operator learning methods. All methods developed in this thesis are publicly available in it. More information can be found at tri16.

The following works used TRIOSlib to implement their experiments and methods.

- Stoffalette João, R.. "Projeto de operators de imagens binárias usando combinação de classificadores". In: Master thesis, advisor: Carlos da Silva Santos.

- Dornelles, M. M. ; Hirata, N. S.T. . "Selection of Windows for W-Operator Combination from Entropy Based Ranking". In: 2015 28th SIBGRAPI Conference on Graphics, Patterns and Images (SIBGRAPI), 2015.

- Silva, R. R. ; Montagner, I. S. ; Hirata, N. S. T. . "Remoção de compasso usando combinações de operadores heurísticos e treinados automaticamente". In: Conference on Graphics, Patterns and Images (SIBGRAPI), 2016, Workshop of Undergraduate Works. 
This work and its earlier states were presented in the events listed below.

1. Conference on Graphics, Patterns and Images (SIBGRAPI) 2016;

2. International Conference on Image Processing (ICIP) 2016;

3. Machine Learning Summer School (MLSS) - Arequipa 2016;

4. Machine Learning Summer School (MLSS) - Kyoto 2015;

5. International Computer Vision Summer School (ICVSS) 2015;

6. International Conference on Image Processing (ICIP) 2014;

7. International Conference on Pattern Recognition (ICPR) 2014.

8. Conference on Graphics, Patterns and Images (SIBGRAPI) 2012; 


\section{Chapter 2}

\section{Fundamentals of linear models in Machine Learning}

Machine Learning (ML) is concerned with learning dependency relationships between variables using a set of observations. For instance, given an image of a printed character, we would like to identify it correctly. The identified character, called a label or class, depends on the joint values of all pixels in the image. Each pixel is a feature or variable. Frequently, the target must be learned from a limited number of observations (or using observations that may not be representative sample of the true population).

Let $y$ be a random variable and $P(x)$ be a probability distribution on a random vector $x \in \mathbb{R}^{M}$. Given a set of i.i.d. observations $\left\{\left(x_{i}, y_{i}\right)\right\}_{i=1}^{N}$, with $x_{i}$ taken from probability distribution $P(x)$ and $y_{i}$ taken from probability distribution $P(y \mid x)$, we would like to estimate $y$ from $x$ given that $P(x)$ and $P(y \mid x)$ are unknown.

Let the hypothesis space $\mathcal{H}=\left\{g(x ; \theta), x \in \mathbb{R}^{M}\right\}$ be a family of functions parametrized by $\theta$. The risk functional (Eq 2.1), defined in Vap13, quantifies how well a hypothesis $g(x ; \theta) \in \mathcal{H}$ approximates $y$ based on a loss function $L(\cdot, \cdot)$. Recall that $P(x, y)=P(y \mid x) P(x)$.

$$
R(\theta)=\int L(y, g(x ; \theta)) d P(x, y)
$$

The best approximation of $y$ in $\mathcal{H}$ is parametrized by the solution $\hat{\theta}$ of the risk minimization problem (Eq. 2.2.

$$
\min _{\theta} \int L(y, g(x ; \theta)) d P(x, y)
$$

In this text we are concerned with problems where $y$ assumes a discrete and finite set of values, each one representing a class of equivalence in $\mathbb{R}^{M}$. A natural choice for $L(\cdot, \cdot)$ in this case is the $0-1$ loss, defined below. 
Minimizing the risk functional is equivalent, in this context, to minimizing the probability of making a mistake $(g(x ; \theta) \neq y)$.

$$
L(y, g(x ; \theta))= \begin{cases}1 \text { if } & g(x) \neq y \\ 0 \text { if } & g(x)=y\end{cases}
$$

Since distribution $P(x, y)$ is unknown, we approximate the risk functional(Eq. 2.1) by the empirical risk

$$
R_{e m p}(\theta)=\frac{1}{N} \sum_{i=1}^{N} L\left(y_{i}, g\left(x_{i} ; \theta\right)\right)
$$

which evaluates the risk on the training set $\left\{\left(x_{i}, y_{i}\right)\right\}_{i=1}^{N}$. Remember that $x_{i}$ forms an i.i.d. sample from $P(x)$ and $y_{i}$ were taken from $P(y \mid x)$.

A good solution to the problem of minimizing the empirical risk must have two properties. First, it must have a relatively small value for $R_{e m p}(\theta)$, which means that it approximates $y$ well. Second, it must generalize to unseen data, which means that $R_{e m p}(\theta) \approx R(\theta)$.

We exemplify these two concepts in Figure 2.1. Suppose we are estimating a polynomial $f: \mathbb{R} \rightarrow \mathbb{R}$ of degree 3 (red line) and have a sample of 10 points. Each sample may include a small observation error. There is a unique polynomial of degree 9 that fits all points with zero error, achieving perfect approximation of $y$ in the sample (blue line). However, the degree 9 polynomial is also approximating the errors as it tries to fit all points exactly, resulting in poor generalization. This phenomenon is called overfitting and it usually happens when fitting complex models to small amounts of data. A polynomial of degree 3 (green line) that only approximates the data, but does not fit any point exactly, generates a result much closer to the original function.

Although minimizing the number of mistakes is our desired goal, frequently it is more convenient to work with loss functions $\ell(\cdot, \cdot)$ that only approximate $L(\cdot, \cdot)$ but allow efficient search on the hypothesis space $\mathcal{H}$. A model is, then, characterized by the choice of a hypothesis space $\mathcal{H}$ and a loss function $\ell(\cdot, \cdot)$. In order to obtain the parameters $\theta$ that best approximate $f$ with regard to $\ell(\cdot, \cdot)$ we solve the following problem, which replaces the empirical risk (Eq. 2.4) by its approximation using $\ell(\cdot, \cdot)$.

$$
\min _{\theta} c(\theta)=\frac{1}{N} \sum_{i=1}^{N} \ell\left(y_{i}, g\left(x_{i} ; \theta\right)\right)
$$

Different loss functions result in different optimal solutions for the same $\mathcal{H}$. Typically, the choice of a loss function aims to induce certain properties (sparsity, magnitude of $\theta$, etc) on the solution of Eq. 2.5. Generalization is also affected by the loss $\ell(\cdot, \cdot)$, with some losses being more prone to overfitting than others [HTF09]. 


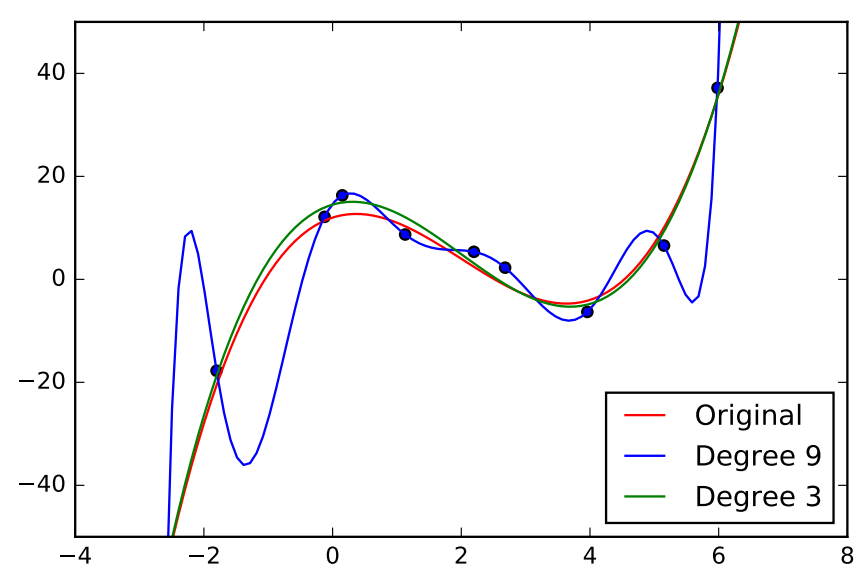

Figure 2.1: The polynomial of degree 9 approximates the data perfectly, but does not generalize. The simpler model has poorer approximation, but follows the original function much closer. This figure was inspired by AM]

We can estimate the "true" risk (Eq. 2.1) of a solution by computing the empirical risk on a second sample to be used as test set. This set must be never seen during training nor used to improve the current solution. The empirical risk on the test set is used to compare the performance of different models for a given task.

Linear models are a very simple but effective class of classification models whose hypothesis space $\mathcal{H}$ is formed by decision functions with linear decision boundaries (i.e. $g(x ; w)=\operatorname{sign}\left(w^{T} x\right)$ ). The weight vector $w$ is the normal vector of a separating hyperplane that discriminates between two classes. In this text we focus on two popular linear models: Logistic Regression and Support Vector Machines.

\subsection{Logistic Regression}

Let $x$ be a random vector of length $M$ and $y$ a discrete random variable taking values in $\{0,1\}$. The probability distribution $P(y \mid x)$ characterizes the dependency of $y$ on the values of $x$. We build the following decision function from $P(y \mid x)$ in order to output a discrete value.

$$
g(x)=\left\{\begin{array}{l}
1 \text { if } \quad P(y=1 \mid x)>0.5 \\
0 \text { otherwise }
\end{array}\right.
$$

If the original distribution $P(y \mid x)$ were known, this decision would minimize the risk functional from Eq. 2.1. The Logistic Regression HTF09] 
model aims to use linear models to characterize $P(y \mid x)$.

Clearly, one cannot just set $P(y=1 \mid x)=w^{T} x$ for some $w \in \mathbb{R}^{M}$, as this assignment does not fulfill all conditions to make $P(y \mid x)$ a probability distribution. We could, however, write the log-odds ratio of $P(y=0 \mid x)$ and $P(y=1 \mid x)$ as a linear function, where log denotes the natural logarithm.

$$
\log \frac{P(y=1 \mid x)}{P(y=0 \mid x)}=w^{T} x, w \in \mathbb{R}^{M}
$$

In this case, probabilities $P(y \mid x)$ are given by

$$
\begin{aligned}
& P(y=1 \mid x ; w)=\frac{e^{w^{T} x}}{1+e^{w^{T} x}} \\
& P(y=0 \mid x ; w)=\frac{1}{1+e^{w^{T} x}} .
\end{aligned}
$$

If $w^{T} x \geq 0$, then $P(y=1 \mid x) \geq \frac{1}{2}$. Therefore, the decision function of the logistic classifier can be rewritten as

$$
g(x ; w)=\left\{\begin{array}{l}
1 \text { if } w^{T} x \geq 0 \\
0 \text { otherwise }
\end{array}\right.
$$

Although the probabilities $P(y \mid x)$ are not linear functions themselves, they induce a linear decision function. Given a weight vector $w \in \mathbb{R}^{M}$, logistic regression defines a probability distribution $P(y \mid x ; w)$ and a linear decision function $g(x ; w)$.

Given a training set $\left\{\left(x_{i}, y_{i}\right)\right\}_{i=1}^{N}, x_{i} \in \mathbb{R}^{M}, y_{i} \in\{0,1\}$, logistic regression models are usually fit using the maximum likelihood principle [HTF09. In this framework the optimum parameter $w^{*}$ is the one that maximizes the likelihood of the training data belonging to the distribution $P(y \mid x ; w)$. This is formalized as the optimization problem in Eq. 2.10.

$$
\max _{w \in \mathbb{R}^{M}} \prod_{i=1}^{N} P\left(y=y_{i} \mid x_{i} ; w\right)
$$

In practice, it is more convenient to minimize the log-likelihood instead (Eq. 2.11). A method to solve to this problem is shown in [HTF09].

$$
\min _{w \in \mathbb{R}^{M}}-\sum_{i=1}^{N} y_{i} w^{T} x_{i}-\log \left(1+e^{w^{T} x_{i}}\right)
$$

Logistic regression models have two advantages when compared to more complex models. First, they provide a confidence on each classification on the form of $P(y=1 \mid x)$. A close decision has $P(y=1 \mid x) \approx 0.5$, while a confident one has $P(y=1 \mid x) \approx 0$ or $P(y=1 \mid x) \approx 1$. Second, the weights $w$ can also reveal important information about the data. For instance, if 


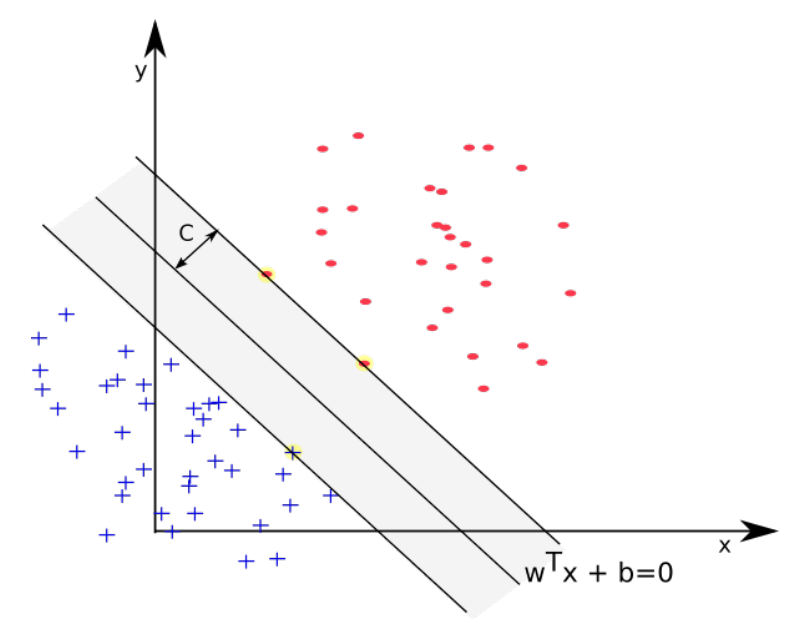

Figure 2.2: The optimal separating hyperplane $w$ is the one that maximizes the margin $C$ between the hyperplane and the closest training instances.

$w_{j} \approx 0$ then feature $x_{j}$ contributes very little to the decision and may be redundant.

The main disadvantage of logistic regression is the simplicity of the decision function, as linear functions may not be sufficient to discriminate complex patterns.

\subsection{Support Vector Machines}

Logistic Regression computes a separating hyperplane by estimating the conditional probabilities $P(y \mid x)$ and building a linear decision function that minimizes the probability of error. Support Vector Machines VVap13, on the other hand, find a linear decision function by explicitly searching for a hyperplane that separates the training data.

We first examine the case where the training data $\left\{\left(x_{i}, y_{i}\right)\right\}, x_{i} \in$ $\mathbb{R}^{M}, y_{i} \in\{-1,1\}$ are linearly separable. We define the optimum separating plane as the one that maximizes the distance between the decision boundary and the nearest training samples. See Figure 2.2 for a illustration of the maximum margin property.

The signed distance between a point $x \in \mathbb{R}^{M}$ and a hyperplane with normal vector $w \in \mathbb{R}^{M}$ is defined as $\frac{w^{T} x}{\|w\|}$. Note, also, that $y_{i}\left(w^{T} x_{i}\right)$ is positive if the signals of $y_{i}$ and $w^{T} x_{i}$ are the same and negative otherwise. Thus, supposing $w$ has unit norm, $y_{i}\left(w^{T} x_{i}\right)$ is the unsigned distance between point $x$ and the hyperplane.

Given a hyperplane $w \in \mathbb{R}^{M},\|w\|=1$, let the margin $C \in \mathbb{R}=$ $\min \left\{y_{i}\left(w^{T} x_{i}\right)\right\}$ be the smallest distance between $w$ and the training samples $\left\{x_{i}, y_{i}\right\}$. Clearly, $y_{i}\left(w^{T} x\right) \geq C, i=1, \ldots, N$. Since we suppose that data is 
linearly separable, there is a vector $w$ that satisfies these constraints. The optimal separating hyperplane $w^{*}$ is the one that satisfies all constraints with the largest margin $C$. This is formalized in the following optimization problem.

$$
\begin{array}{ll}
\max _{C \in \mathbb{R}, w \in \mathbb{R}^{M}} C \text { s.t. } & \\
& y_{i}\left(w^{T} x_{i}\right) \geq C, i=1, \ldots, N \\
& \|w\|=1
\end{array}
$$

We can drop the constraint $\|w\|=1$ by replacing the constraints in Eq. 2.12 with

$$
y_{i}\left(w^{T} x_{i}\right)>C\|w\|, i=1, \ldots, N .
$$

Finally, since any positive multiple of $w$ also satisfies all constraints, we can arbitrarily set $\|w\|=\frac{1}{C}$. This results in the constraints $y_{i}\left(w^{T} x_{i}\right) \geq 1$ and objective $\frac{1}{\|w\|}$. This transformed problem can be more conveniently expressed as below.

$$
\begin{array}{ll}
\min _{w \in \mathbb{R}^{M}} & \frac{1}{2}\|w\|^{2} \text { s.t. } \\
& y_{i}\left(w^{T} x_{i}\right) \geq 1, i=1, \ldots, N .
\end{array}
$$

By examining the first order optimality conditions of Eq. 2.14 (more widely known as KKT conditions [WN99]), we can derive a maximization problem on a different set of variables that has the same optimal value as Eq. 2.14 and such that its variables are related to the original ones. This maximization problem is the dual of the original problem (called the primal problem). The dual of Eq. 2.14 is presented below, where $X \in \mathbb{R}^{N \times M}$ stores one example $x_{i}$ per row.

$$
\begin{aligned}
& \max _{\alpha \in \mathbb{R}^{N}} \sum_{i=1}^{N} \alpha_{i}-\frac{1}{2}(y \alpha)^{T} X X^{T}(y \alpha) \text { s.t. } \\
& \sum_{i=1}^{N} y_{i} \alpha_{i}=0 \\
& \alpha \geq 0, i=1, \ldots, N
\end{aligned}
$$

Each dual variable $\alpha_{i}$ is associated with a primal constraint $y_{i}\left(w^{T} x_{i}\right) \geq 1$. The optimality conditions of Eq. 2.14 state that if a primal constraint is saturated (i.e. satisfied with equality) then its corresponding $\alpha_{i}$ is larger than 0 . If a primal constraint is not saturated, then $\alpha_{i}=0$. In geometrical terms, a saturated constraint indicates that the example $x_{i}$ lies on the margin of the decision function. 
Given the optimal dual solution $\alpha * \in \mathbb{R}^{N}$, the solution to the primal problem is given by

$$
w^{*}=\sum_{i=1}^{N} \alpha_{i}^{*} y_{i} x_{i} .
$$

The primal solution $w$ depends only on the examples $x_{i}$ that lie on the margin, as all other examples have $\alpha_{i}=0$. Thus, although we need all training examples to compute the solution, only a small portion actually defines the solution of the problem. Each training sample $x_{i}$ such that $\alpha_{i}>0$ is called a support vector.

Eq. 2.14 does not have a solution if training data is not linearly separable, as it is impossible to satisfy all constraints simultaneously. We can adapt it to allow some training samples to be inside (or at the wrong side of) the margin by adding a non-negative slack variable $\xi_{i} \geq 0, i=1, \ldots, N$ for each sample and adopting the relaxed constraints

$$
y_{i}\left(w^{T} x_{i}\right) \geq 1-\xi_{i}, i=1, \ldots, N .
$$

All constraints can be satisfied with a large enough $\xi_{i}$. Thus, we would like to have as many $\xi_{i}=0$ as possible. We do this by adding the sum of all $\xi_{i}$ to the cost, resulting in the soft-margin $S V M$ (Eq. 2.18). The $\eta>0$ constant controls (roughly) the number of $\xi \neq 0$ and, thus, impacts directly the number of constraints that are allowed to be violated.

$$
\begin{array}{ll}
\min _{w \in \mathbb{R}^{M}} & \frac{1}{2}\|w\|^{2}+\eta \sum_{i=1}^{N} \xi_{i} \text { s.t. } \\
& y_{i}\left(w^{T} x_{i}\right) \geq 1-\xi_{i}, i=1, \ldots, N . \\
& \xi_{i} \geq 0
\end{array}
$$

The introduction of the slack variables $\xi$ adds the constraint $\alpha_{i} \leq \eta$ for all $i=1, \ldots, N$. The dual is, then, given by

$$
\begin{aligned}
\max _{\alpha \in \mathbb{R}^{N}} & \sum_{i=1}^{N} \alpha_{i}-\frac{1}{2}(y \alpha)^{T} X X^{T}(y \alpha) \text { s.t. } \\
& \sum_{i=1}^{N} y_{i} \alpha_{i}=0 \\
& 0 \leq \alpha \leq \eta, i=1, \ldots, N
\end{aligned}
$$

As in the separable case, this problem is simpler to solve than its primal problem (Eq. 2.18) and can also be solved by standard methods WN99. The decision function is given by 


$$
g(x ; w)=\sum_{i=1}^{N} y_{i} \alpha_{i} x_{i}^{T} x
$$

\subsection{Nonlinear decisions using feature maps}

Linear methods are a simple and convenient way of representing decision boundaries for classification problems. As seen in the last section, the resulting optimization problems can be efficiently solved and the resulting weights can be interpreted as the importance of each feature. However, it is unlikely that the true decision function $f(x)$ is a linear model. Instead of using completely nonlinear methods, such as Neural Networks, we can generate nonlinear decision boundaries using linear methods by doing a basis expansion of the feature vector $x$.

Let $\phi: \mathbb{R}^{M} \rightarrow \mathbb{R}^{L}$ be a feature map that augments the original feature vector $x \in \mathbb{R}^{M}$ with new components derived from $x$. For instance, each component of $\phi(x)$ may result from the application of some nonlinear function in $x$. The decision function

$$
g(x ; w)=\operatorname{sign}\left(w^{T} \phi(x)\right)
$$

is nonlinear in the original feature space. However, it is a linear decision in $\mathbb{R}^{L}$. Thus, we can estimate linear boundaries in $\mathbb{R}^{L}$, using the techniques presented in the previous chapter, that result in nonlinear boundaries in $\mathcal{X}$. The only additional work is to compute $\phi(x)$ for every element $x_{i}$ of the training set and compute $\phi(x)$ during classification.

Choosing adequate $\phi(x)$ is also a concern when using basis expansions. This can be used to introduce prior knowledge about the input space $\mathbb{R}^{M}$. Many types of features can be created using this approach, the most popular being polynomial features, interaction terms combining the output of many original features and splines [HTF09. The use of basis expansions in SVMs is explored in more detail in Chapter 4 


\section{Chapter 3}

\section{Fundamentals of W-operator learning}

Let $\mathbb{E} \subset \mathbb{Z}^{2}$ be the discrete Euclidean grid and $\kappa=\{0,1, \ldots, k-1\}$ a set of gray levels. A gray-level image is a function $I: \mathbb{E} \rightarrow \kappa$ and a binary image is a function $b: \mathbb{E} \rightarrow\{0,1\}$. Clearly, the set of all binary images is contained in the set of gray-level images. The set of all gray-level images is denoted $\kappa^{\mathbb{E}}$ and the set of all binary images is denoted $\{0,1\}^{\mathbb{E}}$. We use the letters $I, O$ for images and $p, q \in \mathbb{E}$ for points in $\mathbb{E}$.

Binary images can also be represented using set notation. An image is a subset of $\mathbb{E}$ and the set of all images is the power set $\mathcal{P}(\mathbb{E})$. Both representations are equivalent. A binary image $b$ can also be represented in set notation as $B=\{p \in \mathbb{E} \mid b(p)=1\}$. The inverse is also possible: a binary image $B \subseteq \mathbb{E}$ in set notation is represented in function notation as

$$
b(p)=\left\{\begin{array}{l}
1 \text { if } p \in B \\
0 \text { otherwise }
\end{array}\right.
$$

An image operator is a function $\Psi: \kappa^{\mathbb{E}} \rightarrow \kappa^{\mathbb{E}}$ that takes as input one image and outputs another image. Complex image operators are usually built by combining basic operators such as dilations, erosions and convolutions [GW06].

Two important properties of image operators are Translation Invariance and Local Definition [Hei94]. An operator $\Psi$ is translation invariant (TI) if

$$
\Psi(I)_{p}=\Psi\left(I_{p}\right)
$$

where $I_{p}(q)=I(q-p), q, p \in \mathbb{E}$. Operator $\Psi$ is locally defined (LD) inside a finite window $W \subset \mathbb{E}$ if

$$
\Psi(I)(p)=\Psi\left(\left.I\right|_{W_{p}}\right)
$$


where $\left.I\right|_{W}$ is the restriction of the domain of $I$ to the points in $W$. Therefore, $\Psi(I)(p)$ is computed using only the pixels in the neighborhood defined by $W$ centered at $p$.

An image operator $\Psi$ is a $W$-operator Hei94, BB91 if and only if it is both TI and LD. These two properties imply that there exists a local function $\psi: \kappa^{W} \rightarrow \kappa$ such that

$$
\Psi(I)(p)=\psi\left(\left.I_{-p}\right|_{W}\right)
$$

for all $I \in \kappa^{\mathbb{E}}, p \in \mathbb{E}$. Every $W$-operator is uniquely characterized by its local function $\psi$ [Hei94]. For this reason $\psi$ is also called the characteristic function of $\Psi$.

Every $\psi$ can be written in a canonical form [BB91, BB91] that is defined using a small family of mathematical morphology operators. However, representing operators in this form is usually not practical: a very large number of basic operators is necessary to represent complex operators. Thus, we do not explore this representation further in this text.

We restrict our analysis to $W$-operators with binary output. Although this definition may seem too restrictive, many image processing tasks can be represented as binary segmentation problems, which means that their output is a binary image. Therefore, from now on we consider $\psi: \kappa^{E} \rightarrow\{0,1\}$.

\subsection{Learning formulation}

Given pairs of images $\left\{\left(I_{i}, O_{i}\right)\right\}$ such as the ones shown in Figure 1.1, we would like to estimate a $W$-operator $\Psi$ such that $\Psi\left(I_{i}\right) \approx O_{i}$. Since $\Psi$ is entirely characterized by its function $\psi: \kappa^{W} \rightarrow\{0,1\}$, the learning task is reduced to estimating $\psi$ instead of $\Psi$. Many earlier works Hir09, DH15, BTHH00 have explored a purely morphological approach to try and learn $\psi$ in its canonical or basis form. We present here an alternative formulation that allows representations taking continuous values and that does not enforce the representation of image data using lattices. This approach favors the use of traditional Machine Learning techniques based on continuous optimization methods. It also has practical advantages over a pure mathematical morphology approach, as continuous optimization is often regarded as simpler than discrete optimization. For more information on the mathematical morphology aspect of $W$-operator learning see [BTHH00].

The characteristic function $\psi$ can be viewed as a pixel classifier that takes as input $|W|$ features and outputs either black or white. The TI and LD properties imply that the value of every output pixel is computed independently and uses only the pixels inside $W$ in the input. The features are extracted by positioning $W$ over every pixel in the input images and copying the values to a flattened array, which is then classified to obtain the output value for that pixel. This process in summarized in Figure 3.1 . 


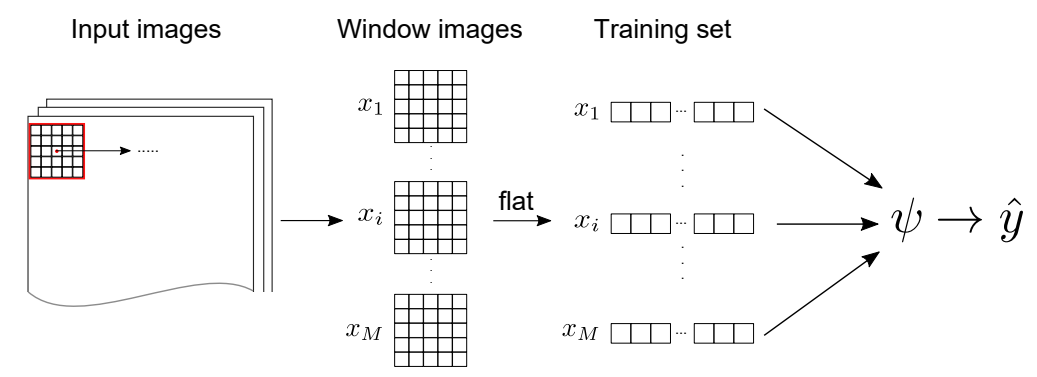

Figure 3.1: Summary of the learning process for $W$-operators.

The learning process for estimating $\psi$ is formalized as follows BTHH00, Hir09]. Suppose that images follow a jointly stationary process $(I, O)$. Then we would like to estimate a $W$-operator $\Psi$ such that we minimize the expected Mean Absolute Error (Eq 3.4). Since $\Psi$ outputs only 0 or 1 for each pixel, minimizing the MAE is equivalent to minimizing the expected error of $\Psi$.

$$
M A E\langle\Psi\rangle=E(|\Psi(I)-O|)
$$

Since $(I, O)$ is jointly stationary and $\Psi$ is a $W$-operator defined by a window $W$, we can describe the application of $\Psi$ in $(I, O)$ using a local process $(X, y)$, where $X \in \kappa^{|W|}$ is a random vector representing image patches observed by $W$ in input images $I$ and $y \in\{0,1\}$ are the corresponding binary outputs in $O$. Then we can rewrite $\mathrm{Eq} 3.4$ in terms of the characteristic function $\psi$ and the local process $(X, y)$ as below.

$$
M A E\langle\Psi\rangle=E(|\psi(X)-y|)
$$

Since the minimization of Eq 3.4 can be done by minimizing Eq. 3.5 , it is sufficient to learn the characteristic function $\psi$ in order to learn the $W$-operator $\Psi$. In practice the local process $(X, y)$ is not known, but it can be estimated from data. Given a set of training images $\left\{\left(I_{l}, O_{l}\right)\right\}_{l=1}^{L}$, for each pixel $p$ in all images we record the observed pattern $x_{i} \in \kappa^{|W|}$ under window $W$ and its corresponding output $y_{i} \in\{0,1\}$. Then we approximate the MAE by the Empirical Mean Absolute Error(Eq 3.6) using a sample $\left\{\left(x_{i}, y_{i}\right)\right\}_{i=1}^{N}$ of local process $(X, y)$.

$$
M A E\langle\Psi\rangle \approx \frac{1}{N} \sum_{i=1}^{N}\left|\psi\left(x_{i}\right)-y_{i}\right|
$$

We can minimize Eq. 3.6 by assigning to each observed pattern $x$ a label according to Eq. 3.7. The probabilities $P(1 \mid x)$ and $P(0 \mid x)$ are computed from the training examples $\left(x_{i}, y_{i}\right)$. 


$$
\psi(x)= \begin{cases}1 & P(1 \mid x) \geq P(0 \mid x) \\ 0 & \text { otherwise }\end{cases}
$$

Note that Eq. 3.7 only assigns labels to the observed patterns. An additional step is needed in order to fully define $\psi$. Therefore, we can divide the learning process for $W$-operators in three steps:

1. Feature Extraction Slide window $W$ over all pixels from images $\left\{\left(I_{l}, O_{l}\right)\right\}_{l=1}^{L}$ and record patterns $\left\{\left(x_{i}, y_{i}\right)\right\}_{i=1}^{N}$

2. Error minimization Assign a label $\hat{y}_{i}$ to all observed patterns $x_{i}$. This may be done according to Eq. 3.7 .

3. Generalization Assign a label to unobserved patterns.

ML algorithms typically do steps 2 and 3 at the same time during the estimation of a classifier's parameter set. Therefore, in this text we divide the learning process in only two steps: Feature Extraction and Training. We also expand the first step to include a function $\phi: \kappa^{|W|} \rightarrow \mathbb{R}^{M}$ that transforms the raw pixel data into a convenient representation. This $\phi$ can be either defined manually or learned from data. The second phase (Training) consists in learning a classifier $g: \mathbb{R}^{M} \rightarrow\{0,1\}$ that outputs the final pixel value. The $W$-operator is then characterized by the composite function $\psi=$ $g(\phi(x))$. The traditional approach used in many earlier works MHH14b, Hir09, MCB06, DH15, dSdS10 sets $\phi(x)=x$ in the Feature Extraction step and uses the ISI algorithm [HRB07] in the Training step. Gray-level operators used Decision Trees in the training step [HBBD06].

This two-step process allows us to compute higher-level features from the window image and use simpler models for classification. For instance, Aperture operators HBBD06, which position a window also on the graylevel, can be explained as a feature extraction function $\phi(x)=\max (\min (x-$ $\left.\left.x_{c}, k\right),-k\right)$, where $c$ is the index of the center $W$. We also present a similar decomposition for two-level operators [Hir09] in the next chapter. We can also easily apply traditional ML techniques in the context of $W$-operator learning. As presented in Sections 4 and 5 , these techniques frequently have a visual interpretation when applied to image data.

The window $W$ chosen has a significant impact on the performance of $\psi$, as it defines the feature space used in $g$. Choosing a suitable window for each task is an important step of $W$-operator learning. Small windows cannot discriminate spatially large patterns. They generate many observations $\left(x_{i}, y_{i}\right)$ such that $P\left(1 \mid x_{i}\right) \approx P\left(0 \mid x_{i}\right) \approx 0.5$. Thus, they result in operators with poor performance. On the other hand, using large $W$ requires very large training sets. Since training data size is usually limited this also results in operators with poor performance. Successfully training $W$ operators 
requires balance between discriminating power (window size) and generalization performance. The feature extraction function $\phi$ plays an important role in this balance. If it is learned from data, we can focus on making the decision function simpler, which hopefully means better generalization. If defined manually, $\phi$ can introduce a priori knowledge not observed in the training data. This is specially useful when dealing with small training sets. Thus, setting $\phi$ adequately allows us to use larger windows and better utilize training data.

\subsection{Two level operators}

Two-level operator design Hir09 is a technique to learn $W$-operators over large windows. It consists in training several operators using windows with moderate size and combining their results into a single image. The combination is done using stacked generalization [Wol92]. Although no operator is trained using a large window, the result depends on the union of all first level windows. Thus, each pixel in the combined output depends on a large window in the original input image.

Let $\left\{\psi_{j}\right\}_{j=1}^{m}$ be a set of $W$-operators trained with windows $W_{j}, j=$ $1, \ldots, m$ and $D=\cup_{j=1}^{m} W_{j}$ a domain window. A second level pattern $z \in$ $\{0,1\}^{m}$ is defined as the concatenation of the outputs of $\psi_{j}$ for a given input pattern $x \in \kappa^{D}$. We can compute $z$ by applying the following feature transform $(\mathrm{Eq} 3.8)$.

$$
z=\phi(x)=\left(\psi_{1}\left(\left.x\right|_{W_{1}}\right), \ldots, \psi_{m}\left(\left.x\right|_{W_{m}}\right)\right) .
$$

The second-level operator or combination operator $\psi^{(2)}:\{0,1\}^{m} \rightarrow$ $\{0,1\}$ is a $W$-operator that takes as input a second-level pattern and outputs a pixel value. Since it uses information in the neighborhood defined by $D, \psi^{(2)}$ is actually defined over a large window. Frequently we refer to a two-level operator by $\psi^{(2)}$ even though it is actually defined as $\psi(x)=\psi^{(2)}(z)=\psi^{(2)}(\phi(x))$.

Two-level operators are estimated in a two-step process. In the first step we train operators $\left\{\psi_{j}\right\}$ on image set $T_{1}$ using windows $W_{j}, j=1, \ldots, m$, respectively, and apply all $\psi_{j}$ to the images from a second image set $T_{2}$. In the second step we form a second level training set $\left\{\left(z_{i}, y_{i}\right)\right\}_{i=1}^{N}, z_{i} \in\{0,1\}^{m}$, where $z_{i}$ is assembled using the resulting images from the previous step. Then we can estimate $\psi^{(2)}$ using the same method as the individual operators $\psi_{j}$. This process is illustrated in Figure 3.2 .

Choosing which operators to combine is an important step in two-level learning, as they define the feature space computed by $\phi$ and used in the decision made by $\psi^{(2)}$. As with classifier ensembles [Kun04, a "good" set of firstlevel operators should have some diversity in the sense that their decisions 


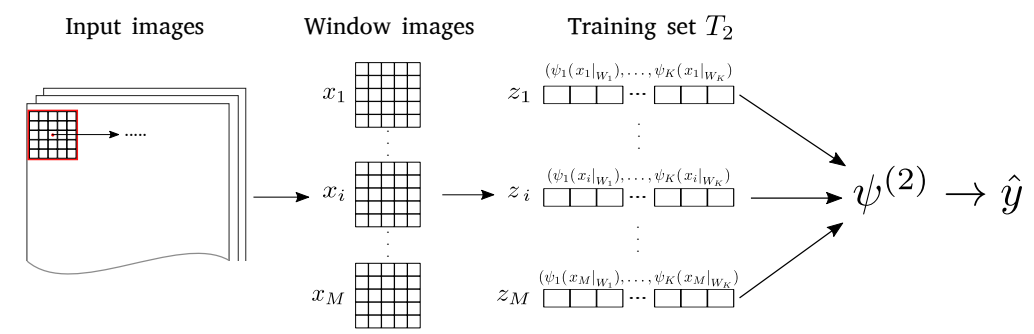

Figure 3.2: Illustration of the learning process for second-level operators.

should coincide in most but not all patterns. Since $T_{1}$ is fixed, choosing operators usually means choosing which windows to use in each operator. Previous works [Hir09, DH15] propose combining windows with basic shapes, such as rectangles and lines, covering a large domain window. These manually determined combinations achieve superior results to operators trained directly using large windows. Automatically selecting windows is a subject of study of many works [MHH16, MHH14b, DH15, SHH10, MCB06]. We present a summary of these techniques in Section 5.1 . 


\section{Chapter 4}

\section{Support Vector Machines applied to $W$-operator learning}

Support Vector Machines (SVM) have several interesting properties when applied to $W$-operator learning. First, they are known for being resistant to overfitting even in high-dimensional feature spaces. Second, the maximum margin property results in classifiers where most of the examples are far from the decision boundaries. Finally, when using kernels the solution depends on a similarity measure computed on all members of the training set. Thus, by using SVMs it should not be necessary to observe many visually similar patterns to generalize well.

In Section 2.3 we discussed the use of feature maps to compute nonlinear boundaries using linear methods. In this chapter we overview the use of feature maps in SVMs and list under what conditions a symmetric function $k(\cdot, \cdot)$ has a feature map representation. Then, we discuss the feasibility of using kernel SVMs in the context of $W$-operator learning and describe a technique to approximate a kernel's implicit feature map and apply it to learn $W$-operators. Finally, we choose kernels that are adequate for processing either binary or gray-level images and describe their interpretation when applied to image data.

\subsection{Kernel Support Vector Machines}

SVMs are specially suited for basis expansions. First, notice that in the dual soft-margin SVM problem the training examples appear only in inner product form $\left(X^{T} X\right.$ in Eq. 2.19 and $x_{i}^{T} x$ in Eq.2.20). Thus, given a feature 
map ${ }^{1} \phi: \mathcal{X} \rightarrow \mathcal{F}$ that transforms a feature vector $x \in \mathcal{X}$ into a new feature vector $\phi(x) \in \mathcal{F}$ we define a function $k: \mathcal{X} \times \mathcal{X} \rightarrow \mathbb{R}$ such that $k\left(x, x^{\prime}\right)=$ $\phi(x)^{T} \phi\left(x^{\prime}\right)$. Then, we rewrite Eq. 2.19 as below, where $K \in \mathbb{R}^{N \times N}$ is a matrix such that $K_{i j}=k\left(x_{i}, x_{j}\right)$. This formulation is called kernel $S V M$ and matrix $K$ is called the Gram matrix of $k$.

$$
\begin{aligned}
\max _{\alpha \in \mathbb{R}^{N}} & \sum_{i=1}^{N} \alpha_{i}-\frac{1}{2}(y \alpha)^{T} K(y \alpha) \text { s.t. } \\
& \sum_{i=1}^{N} y_{i} \alpha_{i}=0 \\
& 0 \leq \alpha \leq \eta, i=1, \ldots, N
\end{aligned}
$$

A decision is made by computing

$$
g(x ; \alpha)=\operatorname{sign}\left(\sum_{i=1}^{N} \alpha_{i} y_{i} k\left(x_{i}, x\right)\right)
$$

This optimization problem estimates a linear decision boundary in the space $\mathcal{F}$. However, it does not explicitly compute the transformed feature vectors $\phi(x) \in \mathcal{F}$. It is only necessary to know how to compute the function $k(\cdot, \cdot)$, which can be interpreted as a similarity measure between patterns SS01. The bigger the similarity between $x$ and $x_{i}$ the bigger its contribution to the decision (assuming, of course, that $\alpha_{i} \neq 0$ ).

It is natural to wonder, then, for what class of functions $k(\cdot, \cdot)$ Eq. 4.1 can be efficiently solved. In other words, what are the conditions a generic function $k(\cdot, \cdot)$ must satisfy to guarantee that Eq. 4.1 has a solution?

The optimization problem in Eq4.1 is a convex quadratic programming if the matrix $K$ is positive definite [WN99]. Such problems can be efficiently solved using standard optimization methods. A matrix $K$ is positive definite if $x^{T} K x>0$ for all $x \in \mathcal{X}, x \neq 0$.

A function $k: \mathcal{X} \times \mathcal{X} \rightarrow \mathbb{R}$ is a positive definite kernel if, for any set $\left\{x_{1}, \ldots, x_{n}\right\}, x_{i} \in \mathcal{X}$, the matrix $K \in \mathbb{R}^{n \times n}, K_{i j}=k\left(x_{i}, x_{j}\right)$ is positive definite. Thus, we may use any positive definite kernel in Eq. 4.1.

In order to solve Eq. 4.1 it is necessary to compute and store the Gram matrix $K \in \mathbb{R}^{N \times N}$. This is often unfeasible in the context of $W$-operator learning. Since each pixel generates a training sample, training sets contain at least hundreds of thousands of examples. Computing $K$ (or even storing it) may take a very long time. Classification may also be an issue. Kernel SVMs perform classification by first computing the value of $k\left(x, x_{i}\right)$ for all support vectors $\left(\alpha_{i} \neq 0\right)$ and then computing an inner product with $\alpha$. It

\footnotetext{
${ }^{1}$ We use $\mathcal{X}$ as a generic input space and $\mathcal{F}$ as a generic output space. Typically $\mathcal{X}=\mathbb{R}^{M}$ and $\mathcal{F}=\mathbb{R}^{L}, L>M$.
} 
may take a long time to process one image if we repeat this procedure for all pixels in the image. Therefore, naively applying kernel SVMs to $W$-operator leaning is not ideal.

An approach explored in WS01, RR09 is to approximate the kernel $k(\cdot, \cdot)$ by computing a feature map $\phi: \mathcal{X} \rightarrow \mathbb{R}^{m}$ such that $k\left(x, x^{\prime}\right) \approx$ $\phi(x)^{T} \phi\left(x^{\prime}\right)$. Using this approach classification can be made much faster, since it would be linear in $m<<N$. Instead of solving the full kernel SVM (Eq. 4.1 we instead solve the approximation given by Eq. 4.3 .

$$
\begin{aligned}
\min _{w \in \mathbb{R}^{m}} & \frac{1}{2}\|w\|^{2} \text { s.t. } \\
& y_{i}\left(w^{T} \phi\left(x_{i}\right)\right) \geq 1, i=1, \ldots, N
\end{aligned}
$$

\subsection{Approximating a kernel's implicit feature map}

The two most popular methods for estimating the implicit feature map $\phi$ are the Nyström method [WS01] and Random Fourier Features [RR09]. The Nyström method approximates $\phi$ based on the eigendecomposition of a submatrix of the Gram matrix $K$. Random Fourier Features approximate shiftinvariant $\left(k\left(x, x^{\prime}\right)=k\left(x-x^{\prime}\right)\right)$ kernels by sampling from a probability distribution based on a kernel's Fourier decomposition. A comparison between these methods $\left[\mathrm{YLM}^{+} 12\right]$ indicates that the Nyström method achieves results superior to Random Fourier Features, but it is also more expensive to compute.

The Nyström method relies on Mercer's condition for its approximation. Let $T_{k}$ be the operator defined by a symmetric function $k: \mathcal{X} \times \mathcal{X} \rightarrow \mathbb{R}$

$$
\left(T_{k} f\right)(x)=\int_{\mathcal{X}} k\left(x, x^{\prime}\right) f\left(x^{\prime}\right) d x^{\prime}
$$

Mercer's condition [SS01, Vap13 states that, for positive definite kernels there exists an expansion

$$
k\left(x, x^{\prime}\right)=\sum_{j=1}^{\infty} \lambda_{j} \varphi_{j}(x) \varphi_{j}\left(x^{\prime}\right)
$$

where $\varphi_{j}$ are the normalized orthogonal eigenfunctions of $T_{k}$ associated with eigenvalues $\lambda_{j}>0$, sorted in non-increasing order. Since every $\varphi_{j}$ is orthogonal, this implies 


$$
\begin{array}{r}
\left(T_{k} \varphi_{j}\right)(x)=\int_{\mathcal{X}} k\left(x, x^{\prime}\right) \varphi_{j}\left(x^{\prime}\right) d x^{\prime}= \\
\int_{\mathcal{X}}\left[\sum_{k=1}^{\infty} \lambda_{k} \varphi_{k}(x) \varphi_{k}\left(x^{\prime}\right)\right] \varphi_{j}\left(x^{\prime}\right) d x^{\prime}= \\
\sum_{k=1}^{\infty}\left[\lambda_{k} \varphi_{k}(x) \int_{\mathcal{X}} \varphi_{k}\left(x^{\prime}\right) \varphi_{j}\left(x^{\prime}\right) d x^{\prime}\right]= \\
\lambda_{j} \varphi_{j}(x) \int_{\mathcal{X}} \varphi_{j}\left(x^{\prime}\right) \varphi_{j}\left(x^{\prime}\right) d x^{\prime}=\lambda_{j} \varphi_{j}(x),
\end{array}
$$

since the eigenfunctions $\varphi_{j}$ are orthogonal $\left(\int_{\mathcal{X}} \varphi_{k}\left(x^{\prime}\right) \varphi_{j}\left(x^{\prime}\right) d x^{\prime}=0\right.$ if $\left.k \neq j\right)$ and normalized $\left(\int_{\mathcal{X}} \varphi_{j}\left(x^{\prime}\right) \varphi_{j}\left(x^{\prime}\right) d x^{\prime}=1\right)$.

Given a sample $\left\{x_{i}\right\}_{i=1}^{N}$ taken according to probability distribution $p(x)$, we could approximate the integral operator $T_{k} \varphi_{j}$ ) (Eq.4.4 with a weighted summation, which results in

$$
\left(T_{k} \varphi_{j}\right)(x) \approx \frac{1}{N} \sum_{i=1}^{N} k\left(x, x_{i}\right) \varphi_{j}\left(x_{i}\right) \approx \lambda_{j} \varphi_{j}(x) .
$$

Suppose we want to estimate the value of $T_{k} \varphi_{j}$ for all examples from our sample $\left\{x_{i}\right\}_{i=1}^{N}$ and some fixed $j$. We can arrange this in matrix notation as

$$
\lambda_{j}\left(\begin{array}{c}
\varphi_{j}\left(x_{1}\right) \\
\ldots \\
\varphi_{j}\left(x_{N}\right)
\end{array}\right)=\frac{1}{N} K^{(N)}\left(\begin{array}{c}
\varphi_{j}\left(x_{1}\right) \\
\ldots \\
\varphi_{j}\left(x_{N}\right)
\end{array}\right),
$$

where $K^{(N)}$ is the Gram matrix of our sample. Since the eigenfunctions are normalized, we also have the following empirical constraint.

$$
\begin{aligned}
& \frac{1}{N} \sum_{k=1}^{N} \varphi_{i}\left(x_{k}\right) \varphi_{j}\left(x_{k}\right)=0 \text { if } i \neq j \\
& \frac{1}{N} \sum_{k=1}^{N} \varphi_{j}\left(x_{k}\right) \varphi_{j}\left(x_{k}\right)=1
\end{aligned}
$$

Equation 4.8 resembles the definition of the eigenvalues/vectors of $K^{(N)}$. Thus, let

$$
K^{(N)} U^{(N)}=U^{(N)} \Delta^{(N)}
$$

be the eigendecomposition of $K^{(N)}$, where $U^{(N)} \in \mathbb{R}^{N \times N}$ is an orthonormal matrix with eigenvector in its columns and $\Delta^{(N)}=\operatorname{diag}\left(\lambda^{(N)}\right)$. By 
combining Eqs. 4.8 and 4.10 we see that $\lambda_{j} \approx \frac{\lambda_{j}^{(N)}}{N}$. The empirical constraints (Eq. 4.9 are satisfied when we set $\varphi_{j}\left(x_{i}\right)=\sqrt{N} U_{i, j}^{(N)}$. Thus, we can approximate $\lambda_{j}$ and $\varphi_{j}\left(x_{i}\right)$ from our sample using the following expressions.

$$
\begin{array}{r}
\lambda_{j} \approx \frac{\lambda_{j}^{(N)}}{N} \\
\varphi_{j}\left(x_{i}\right) \approx \sqrt{N} U_{i, j}^{(N)}
\end{array}
$$

Finally, we can combine Eq. 4.11 with Eq. 4.7 to determine an approximation for $\varphi_{j}(x)$. Since the sample has size $N$, we can estimate at most the largest $N$ pairs of eigenvalues/functions.

$$
\varphi(x)_{j} \approx \frac{\sqrt{N}}{\lambda^{(N)}} \sum_{i=1}^{N} k\left(x, x_{i}\right) U_{i, j}^{(N)}
$$

Using this expression we can evaluate the kernel at point $k\left(x, x^{\prime}\right)$ as below.

$$
\begin{aligned}
k\left(x, x^{\prime}\right) & \approx \sum_{j=1}^{N} \lambda_{j} \varphi(x)^{T} \varphi\left(x^{\prime}\right)= \\
& =\sum_{j=1}^{N} \frac{\lambda_{j}^{(N)}}{N}\left(\frac{\sqrt{N}}{\lambda_{j}^{(N)}} \sum_{i=1}^{N} k\left(x, x_{i}\right) U_{i, j}^{(N)}\right)^{T}\left(\frac{\sqrt{N}}{\lambda_{j}^{(N)}} \sum_{i=1}^{N} k\left(x^{\prime}, x_{i}\right) U_{i, j}^{(N)}\right) \\
& =\sum_{j=1}^{N} \frac{\lambda_{j}^{(N)}}{N} \frac{N}{\left(\lambda_{j}^{(N)}\right)^{2}}\left(\sum_{i=1}^{N} k\left(x, x_{i}\right) U_{i, j}^{(N)}\right)^{T}\left(\sum_{i=1}^{N} k\left(x^{\prime}, x_{i}\right) U_{i, j}^{(N)}\right) \\
& =\sum_{j=1}^{N} \frac{1}{\lambda_{j}^{(N)}}\left(\sum_{i=1}^{N} k\left(x, x_{i}\right) U_{i, j}^{(N)}\right)^{T}\left(\sum_{i=1}^{N} k\left(x^{\prime}, x_{i}\right) U_{i, j}^{(N)}\right) \\
& =\sum_{j=1}^{N}\left(\frac{1}{\sqrt{\lambda_{j}^{(N)}}} \sum_{i=1}^{N} k\left(x, x_{i}\right) U_{i, j}^{(N)}\right)^{T}\left(\frac{1}{\sqrt{\lambda_{j}^{(N)}}} \sum_{i=1}^{N} k\left(x^{\prime}, x_{i}\right) U_{i, j}^{(N)}\right)
\end{aligned}
$$

Thus, by setting $\phi^{(N)}(\mathbf{x})=\operatorname{diag}\left(\frac{1}{\lambda^{(N)}}\right) U^{(N)^{T}} K_{\mathbf{x}}$, where $K_{\mathbf{x}}=$ $\left(k\left(\mathbf{x}, x_{1}\right), \ldots, k\left(\mathbf{x}, x_{N}\right)\right)^{T}$, we obtain a feature map $\phi^{(N)}: \mathbb{R}^{M} \rightarrow \mathbb{R}^{N}$ such that $k\left(x, x^{\prime}\right) \approx \phi^{(N)}(x)^{T} \phi^{N}\left(x^{\prime}\right)$. Still, this result is not sufficient to train models using Eq. 4.3. Computing $\phi^{(N)}\left(x_{i}\right)$ for all $i=1, \ldots, N$ requires computing all components of $K$, which is impractical.

When training models using Eq. 4.3 we are interested in using only a small subsample of size $m<<N$ to estimate the value of $\phi^{(N)}\left(x_{i}\right)$. We call 
this subsample the approximation set. For convenience, we use the first $m$ points and partition $K^{(N)}$ as follows.

$$
K^{(N)}=\left[\begin{array}{ll}
K_{(m, m)} & K_{(m, N-m)} \\
K_{(N-m, m)} & K_{(N-m, N-m)}
\end{array}\right]
$$

We now compute the eigendecomposition of $K_{(m, m)}=$ $U^{(m)} \operatorname{diag}\left(\lambda^{(m)}\right) U^{(m)}$ and define the feature map $\phi^{(m)}: \mathbb{R}^{M} \rightarrow \mathbb{R}^{m}$ as

$$
\phi(\mathbf{x})^{(m)}=\operatorname{diag}\left(\frac{1}{\sqrt{\lambda}}\right) U^{(m)^{T}} K_{\mathbf{x}}
$$

with $K_{\mathbf{x}}=\left(k\left(\mathbf{x}, x_{1}\right), \ldots, k\left(\mathbf{x}, x_{m}\right)\right)$. Notice that, for the remaining training samples $\left(x_{i}, i>m\right), K_{x_{i}}=K_{(N-m, m)} \bullet=\left(K_{(m, N-m)_{i} \bullet}\right)^{T}$. Also, $K_{(m, m)}^{-1}=$ $U^{(m)} \operatorname{diag}\left(\frac{1}{\lambda^{(m)}}\left(U^{(m)}\right)^{T}\right.$ and

$$
\begin{aligned}
k\left(x, x^{\prime}\right) & \approx \phi^{(m)}(x)^{T} \phi^{(m)}\left(x^{\prime}\right) \\
& =\left(\operatorname{diag}\left(\frac{1}{\sqrt{\lambda}}\right) U^{(m)^{T}} K_{\mathbf{x}}\right)^{T}\left(\operatorname{diag}\left(\frac{1}{\sqrt{\lambda}}\right) U^{(m)^{T}} K_{\mathbf{x}^{\prime}}\right) \\
& =K_{\mathbf{x}}^{T} U^{(m)} \operatorname{diag}\left(\frac{1}{\lambda^{(m)}}\right) U^{(m)^{T}} K_{\mathbf{x}^{\prime}} \\
& =K_{\mathbf{x}} K_{(m, m)}^{-1} K_{\mathbf{x}^{\prime}} .
\end{aligned}
$$

Thus, we can write the approximation $\hat{K}^{(m)}$ of the full Gram matrix $K^{(N)}$ as $\hat{K}=K_{(N, m)} K_{(m, m)}^{-1} K_{(m, N)}$. More importantly, the Gram matrix of the remaining $N-m$ examples is computed by the following formula.

$$
\hat{K}_{(N-m, N-m)}=K_{(N-m, m)} K_{(m, m)}^{-1} K_{(m, N-m)} .
$$

Thus, by building a feature map $\phi^{(m)}(x)$ from a subsample of size $m<<$ $N$ we are able to solve an approximation of the full SVM problem based on Eq. 4.3. Since $m<<N$, this representation is more economic than solving the dual SVM problem (Eq. 2.19p in storage space and computational time.

\subsection{Kernels applied to image data}

Kernels define a measure of similarity between patterns [SS01]. Choosing an adequate kernel for each task is an important step when building kernel SVMs. In the context of $W$-operator learning, kernels may be chosen based on their interpretations when applied to image data.

We chose polynomial kernels (Eq. 4.18) [SS01] to process binary images. Polynomial kernels of degree $d$ have feature maps that compute the product of up to $d$ variables. The decision function in the transformed space is a weighted sum of products of binary features. This resembles the Sum of 


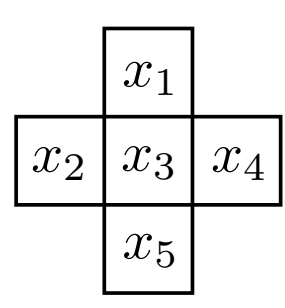

(a) 5 point window

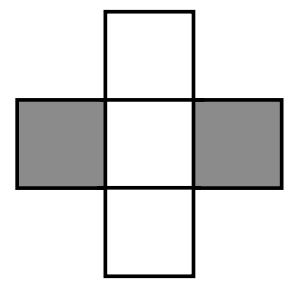

(b) The term $x_{1} x_{3} x_{5}$ detects vertical lines.

Figure 4.1: Products of binary features detect patterns in image data.

Products normal form used in Boolean Function minimization [HRB07]. In terms of binary image data, these products can be understood as binary pattern detectors. We show in Figure 4.1 a visual example of the pattern detection made by polynomial feature maps.

$$
k\left(x, x^{\prime}\right)=\left(x^{T} x^{\prime}+1\right)^{d}
$$

Although these kernels have an explicit feature map representation, it is of very high dimension $\left(\mathcal{O}\left(M^{d}\right)\right.$ for $M$ variables and polynomials of degree d). Thus, using a kernel to compute it is more efficient than expanding the feature set, especially for high-dimensional data. Approximating the polynomial feature map using the Nyström method is also less expensive than using it directly.

We chose Gaussian kernels (Eq. 4.19) to process gray-level images. This kernel addresses several issues present in gray-level patterns. First, it takes into account the relative brightness between patterns, not their absolute value. This can help decrease the number of examples necessary to process images in different illumination conditions. Second, it provides a softthreshold on the similarity between two patterns. Patterns with small differences have values near 1 , while patterns with large differences have values near 0 . Third, the $\gamma$ parameter can be used to tune what "small" and "large" differences mean for each task. Adjusting $\gamma$ is an important step when using this kernel.

$$
k_{\gamma}\left(x, x^{\prime}\right)=e^{-\gamma\left\|x-x^{\prime}\right\|^{2}}
$$

For instance, consider the three window patches shown in Figure 4.2 . With $\gamma=0.01$, as used in Section 7, $k(p 1, p 2)=2.6 e-12, k(p 1, p 3)=$ $3 e-23, k(p 2, p 3)=6.9 e-15$, which means that $p 1$ is more similar to $p 2$ than $p 3$ and that $p 2$ is also more similar to $p 1$ than $p 3$. This agrees with our visual intuition, given that $p 1$ and $p 2$ have large dark gray stripes (vessels in the full image), while $p 3$ is a relatively flat region. Therefore, using a Gaussian kernel to compute the similarity between gray-level patches seems to mimic our visual intuition about image similarity. 

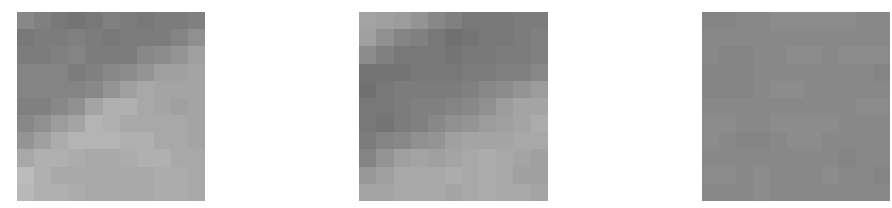

Figure 4.2: Patches $p 1, p 2$ and $p 3$ extracted from a gray-level image. According to a Gaussian kernel (ans visual intuition), $p 1$ is more similar to $p 2$ than $p 3$.

Although Gaussian kernels have a feature map representation of infinite dimension, our experiments show that operators trained with approximations of a Gaussian kernel achieve very good results (see Section 6). Other works [WS01, YLM ${ }^{+}$12, RR09] have reached similar conclusions.

The full KA method is shown below. We choose kernel $k$ according to the task of interest using the criteria explained above. The other hyperparameters $\left(N_{a}, N_{\text {svm }}\right)$ are determined mostly by practical reasons (training time, memory and runtime constraints).

Data: Training sets $T$, Kernel $k$, Approximation size $N_{a}$, SVM training subset size $N_{\text {svm }}$.

Result: Operator $\psi$

$m=N_{a}$

Select $R=\left\{\left(x_{i}^{\prime}, y_{i}^{\prime}\right)\right\}_{i=1}^{m}$ randomly from $T$

$K_{m, m}[i, j]=k\left(x_{i}^{\prime}, x_{j}^{\prime}\right)$

Compute $K_{m, m}=U^{(m)} \operatorname{diag}\left(\lambda^{(m)}\right) U^{(m)}$

Define the feature map $\phi^{(m)}(x)=\operatorname{diag}\left(\frac{1}{\sqrt{\left(\lambda^{(m)}\right)}}\right) U^{(m)^{T}} K_{x}$

Compute $\left.\left\{\phi^{(m)}\left(x_{i}\right), y_{i}\right)\right\}_{i=1}^{N_{s v m}}$.

Solve Eq. 4.3 .

Algorithm 1: KA method 


\section{Chapter 5}

\section{Two-level operator optimization}

Two-level operators combine the output of several $W$-operators trained using moderate size windows, effectively learning a decision function that takes into account a large window. An important step when training twolevel operators is selecting operators to combine. This can be done by selecting a set of windows for training first level operators. The initial approach presented in the original work [Hir09] used rotated and translated versions of basic shapes, such as lines and squares. Subsequent works dSdS10, DH15, MHH14b focused on determining which and how many windows to combine. All these methods follow roughly the same basic algorithm. First they determine, either manually or from data, a set of "good" candidate windows. Then they greedily select which windows/operators to combine. The number of operators in the combination can be determined either using cross-validation or a stopping criteria based on data. These algorithms are very similar to Feature Selection methods in traditional ML scenarios.

Although the combination operator $\psi^{(2)}$ is optimal with regard to the second-level patterns used in the training set, there is no formal notion of an optimal two-level operator. Let $\mathcal{C}_{\psi}=\left\{\psi_{j}\right\}_{j=1}^{2^{M}}$ a set containing first-level operators trained with all subwindows of a domain window $D,|D|=M$. Two-level operator optimization can be thought as finding a subset of $\mathcal{C}_{\psi}$ such that the combination of its operators achieves good performance in both seen and unseen images. We would also like to restrict the number of combined operators in order to avoid the generalization issues found when learning operators using large feature sets. This can be formalized by the following optimization problem, where $\theta$ is the parameter set of $\psi^{(2)}$, $\operatorname{card}\left(\psi^{(2)}\right)$ is the number of operators combined, $\ell\left(\cdot, y_{i} ; \theta\right)$ is a loss function and $\left\{\left(x_{i}, y_{i}\right)\right\}_{i=1}^{N}, x_{i} \in \kappa^{M}, y_{i} \in\{0,1\}$ is a training set extracted using window $D$. 


$$
\begin{aligned}
\min _{\theta} & \sum_{i}^{N} \ell\left(\psi^{(2)}\left(z_{i}\right), y_{i} ; \theta\right) \text { s.t. } \\
& \operatorname{card}\left(\psi^{(2)}\right) \leq K,
\end{aligned}
$$

where $z_{i}$ is a second-level pattern computed using the output of the classifiers in $\mathcal{C}_{\psi}$.

Unfortunately, this optimization problem is NP-hard due to the $\operatorname{card}(\theta) \leq K$ constraint even for very simple models [AK98] and trying all subsets of $\mathcal{C}_{\psi}$ is prohibitive even for small domain windows. Previous works focused on using Feature Selection based approaches to limit the windows used in $\mathcal{C}_{\psi}$ to a small number of "good windows" and then determine $K$ and $\theta$ for this restricted candidate set based on a proxy measure for $\ell$. We present two earlier methods based on this procedure in the next section (5.1). In sequence we present the FS method, a preliminary method we developed in the context of this work. FS uses a Feature Selection based approach that selects first-level windows considering the joint occurrence of all features. In the last section we present a novel approach based on regularized linear models and a specialized optimization algorithm to do operator selection and error minimization at the same time.

\subsection{Previous approaches}

The method presented in [SHH10] (referred here as IT) uses the three-way Interaction Information [McG54] (Eq. 5.2) to group individual points into windows, where $I(Y ; X)$ is the Mutual Information between $Y$ and $X$, where $X$ is a set of independent variables and $Y$ is the dependent variable. In the context of operator learning, $X$ represents the image patches extracted from the input images and $Y$ represent the output pixel value.

$$
I\left(Y ; X_{i} ; X_{j}\right)=I\left(Y ;\left(X_{i}, X_{j}\right)\right)-I\left(Y ; X_{i}\right)-I\left(Y ; X_{j}\right)
$$

Given two variables $X_{i}$ and $X_{j}$, let $\tilde{X}_{j}=\operatorname{XOR}\left(X_{i}, X_{j}\right)$ be a modified variable. The Information Interaction between $Y, X_{i}$ and $\tilde{X}_{j}$ is given by

$$
I\left(Y ; X_{i} ; \tilde{X}_{j}\right)=I\left(Y ; X_{i} ; X_{j}\right)+I\left(Y ; X_{j}\right)-I\left(Y ; \tilde{X}_{j}\right)
$$

If $\tilde{X}_{j}$ carries more information about $Y$ than $X_{j}$ replacing $X_{j}$ by $\tilde{X}_{j}$ causes interaction between variables to decrease in magnitude. Thus, we may obtain more meaningful variables by iteratively decreasing the Interaction Information magnitude and applying the XOR transformation. The parity function (Eq. 5.4) is used to do XOR between already transformed variables.

After some iterations, each transformed variable $\tilde{X}_{j}$ is a XOR of many original variables, We then generate a group from each $X_{j}$ and form windows 
by combining these groups such that the resulting windows are smaller than a predefined number of points.

$$
\operatorname{PAR}(X)= \begin{cases}0 & \text { if } \sum_{j} X_{j} \text { is even } \\ 1 & \text { if } \sum_{j} X_{j} \text { is odd }\end{cases}
$$

Windows are ordered using forward selection based on the maximization of Conditional Mutual Information [Fle04 between the current window/feature and all previously selected features. An ordering $\nu(1), \ldots, \nu(m)$ is computed using Eq. 5.5 .

$$
\begin{gathered}
\nu(1)=\operatorname{argmax}_{j}\left\{I\left(y, z_{\bullet}\right)\right\} \\
\nu(k)=\operatorname{argmax}_{j}\left\{\operatorname { m i n } _ { j < k } \left\{I\left(y ; z_{\bullet k}, z_{\bullet \nu(j)}-I\left(y ; z_{\bullet}(j)\right\}\right\}\right.\right.
\end{gathered}
$$

The number of operators is chosen based on the Minimum Description Length [Grü07](MDL) principle. Given a label vector $y=\left(y_{1}, \ldots, y_{N}\right)$ and a model $M$ parametrized by $\theta$, MDL computes the "length" of $M$ based on the ability of $M$ to faithfully encode the information present in $y$ and the complexity of the parameter set $\theta$. A good model, according to MDL, balances model complexity and predictive power. Given a choice between many models we should, therefore, select the one with the smallest MDL. In IT these principles are encoded as in Eq. 5.6. IT choses the largest number $k$ of operators such that the sequence $L(1) \leq \ldots L(k)$ is decreasing.

$$
L(k)=-\sum_{i=1}^{N}-\log P\left(y_{i} \mid\left(\psi_{1}\left(\left.x_{i}\right|_{W_{1}}\right), \ldots, \psi_{k}\left(\left.x_{i}\right|_{W_{k}}\right)\right)\right)+2^{k}
$$

The WER method (shorthand for Window selection using Entropy based Ranking) [DH15] is also based on information theoretic measures. The conditional entropy of a target variable $\mathbf{y}$ given a random vector $\mathbf{X}$ is defined as

$$
H_{(\mathbf{X}, \mathbf{y})}=-\sum_{x} P(\mathbf{X}=\mathbf{x}) \mathbf{H}(\mathbf{y} \mid \mathbf{X}=\mathbf{x})
$$

If $H_{(\mathbf{X}, \mathbf{y})}$ is small then there is little output confusion. In other words, there are few occurrences where $x_{i}=x_{j}$ and $y_{i} \neq y_{j}$ for some pairs of observations $\left(x_{i}, y_{i}\right)$ and $\left(x_{j}, y_{j}\right)$. This also translates to a small theoretical MAE (Eqs. 3.5 and 3.7) in the training set. According to Eq. 3.7, the optimal operator choses label $y$ for a pattern $x$ when $P(y \mid x)>P\left(y^{\prime} \mid x\right)$ for all other $y^{\prime}$. Thus, a small conditional entropy translates in a small MAE. In fact, minimizing the MAE is equivalent to minimizing the Conditional Entropy.

To accurately estimate $H_{(\mathbf{X}, \mathbf{y})}$ a good estimation of $P(\mathbf{X})$ and $P(\mathbf{y} \mid \mathbf{X})$ is necessary. An earlier work [MCB06] proposed the correcting the conditional 

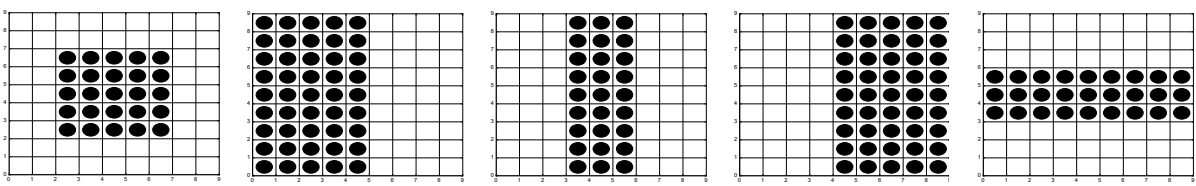

Figure 5.1: Windows used in WER. Black points are used as features in training.

entropy by taking into account how many patterns were not observed. In practice, this resulted in very small windows, as the number of non-observed patterns explodes as window size increases. The authors of [DH15] noticed that, in $W$-operator learning, many patterns are observed only once (which results in $H(\mathbf{y} \mid \mathbf{X}=\mathbf{x})=\mathbf{0}$ ) and most patterns are not observed at all (which results in $P(x)=0)$. They propose the estimation of $H_{(\mathbf{X}, \mathbf{y})}$ by a Corrected Conditional Entropy that assigns a small entropy to patterns observed once. It is defined below,

$$
H_{(\mathbf{X}, \mathbf{y})}^{*}=H^{c} \frac{U}{T}+\sum_{P(\mathbf{X}=\mathbf{x})>\frac{1}{T}} P(\mathbf{X}=\mathbf{x}) \mathbf{H}(\mathbf{y} \mid \mathbf{X}=\mathbf{x})
$$

where $U$ is the number of unique patterns and $T$ is the total number of distinct patterns observed. The authors reason that a single observation of a pattern carries more information than no observation at all and assign a small value $H^{c}$ in this case.

The corrected conditional entropy can be used as a measure of the quality of a given window $W$, as a small $H_{(\mathbf{X}, \mathbf{y})}^{*}$ implies that an operator trained with $W$ will have small MAE.

The WER algorithm consists of the following steps. Given a set of windows $\mathcal{C}=\left\{W_{1}, \ldots, W_{M}\right\}$, it first ranks them according to Eq. 5.8 in a training set $T_{1}$. Then, it trains combinations using the best $j$ windows, for increasing $j$ up to a user defined maximum $J$, in a second training set $T_{2}$. Finally, the best combination size $j^{*}$ is chosen by cross-validation in a validation set.

Instead of computing a different candidate set $\mathcal{C}$ for each problem WER uses manually crafted windows formed by basic shapes, such as lines, squares and rectangles, covering different parts of a large domain window $D$. Some of these windows are shown in Figure 5.1 .

The biggest advantage of WER is that it does not require training operators for all $M$ windows, but only for the best ranked ones. This is very efficient if $J<<M$. On the other hand, WER was proposed for binary operators and may not extrapolate well for gray-level inputs. In this case even for small windows a significant portion of patterns occur only once. This may result in $H^{c} \frac{U}{T}$ being the dominant term of $H_{(\mathbf{X}, \mathbf{y})}^{*}$, which could 
result in a ranking that relies more on the ratio of observed/non-observed patterns than in the output of the optimal operator.

Both IT and WER limit the candidate window set $\mathcal{C}$ to a set of "good" windows that try to ensure diversity in the resulting combination. This also reduces memory and time consumption, since it is not necessary to build $\mathcal{C}_{\psi}$ for a large number of operators. Both also rank first-level operators (though using different measures) and use a sensible way of determining how many operators should be combined (cross-validation for WER, MDL for IT). However, the resulting combination is restricted to those using the selected windows and neither method takes into account the model used to train first and second-level operators.

Both methods were executed on the same datasets as Hir09 and have obtained equal or superior results.

\subsection{Two-level design using feature and window ranking}

The window selection problem can be seen as a feature selection problem. In the case of $W$-operator learning we want to select a subset of variables $W$ (subwindow) from a large set $D$ (domain window) such that $W$ has strong predictive power and uses as little information as possible in order to avoid overfitting. Many feature selection methods are based on feature ranking GGNZ06, which computes a score for each feature and selects the ones with the highest scores. Although these methods are capable of finding features that are individually relevant, they frequently fail to take into account how variables interact GE03. We describe in this section the FS method, a feature selection based method for two-level operator design. It was extensively tested for processing music scores and has obtained state-ofthe-art results in this domain of application. More details on the application of FS to music scores are presented in Section 7.1.

The FS method generates first-level windows using the Relief algorithm [KR92, KRSRP96]. Relief is a feature ranking method that takes into account the co-occurrences between variables. The score $s(j)$ of each component $j$ is based on the relations between a set of prototypes instances and their nearest instances of the same class and of a different class. The algorithm starts with $s(j)=0$ for all $j$ and, for each prototype $p$ updates the score in the following manner, where $p^{+}$is the closest (in euclidean distance) training example of the same class as $p$ and $p^{-}$is the closest training sample of a different class.

$$
s(j)+=-X O R\left(p_{j}, p_{j}^{+}\right)+X O R\left(p_{j}, p_{j}^{-}\right)
$$

The score $s(j)$ of a feature $j$ decreases by 1 when $p_{j} \neq p_{j}^{+}$and $p_{j}=p_{j}^{-}$, which means that feature $j$ hinders discrimination between classes. The 
score $s(j)$ stays the same when $p_{j}=p_{j}^{+}$and $p_{j}=p_{j}^{-}$. In this case feature $j$ neither helps nor hinders classification. Finally, the score increases if $p_{j}=p_{j}^{+}$ and $p_{j} \neq p_{j}^{-}$, which means that feature $j$ can be used to discriminate between classes. Although updates only consider individual features, the interaction between all variables in taken into account by the choice of $p^{+}$and $p^{-}$. Since the euclidean distance depends on the joint occurrence of all features, $s(j)$ also depends indirectly on the interaction between features.

After updating $s(j)$ with respect to all prototypes, the features that help classification are expected to have positive $s(j)$ while confounding features should have large negative $s(j)$. Irrelevant features are expected to have $s(j)$ close to 0 .

A ranking produced by Relief is clearly dependent on which instances are chosen as prototypes. However, the most relevant features should not be heavily affected by this choice, as it is unlikely that the choice of prototypes includes only instances where they are not helpful for classification. The same is expected for the bottom ranked features. Thus, we expect changes in the prototype set to affect more strongly the middle ranked features. We can generate a window from a Relief ranking by selecting only the $M_{R}$ top ranked features.

Relief can be used to generate candidate windows for two-level operators in the same spirit as the basic window shapes from WER or the windows created in IT. By selecting randomly different prototype sets we can generate many different rankings/windows. These windows should have a reasonable intersection, formed by the most relevant features, but should also be diverse, as middle ranked features are expected to be different between rankings.

Let $\mathcal{C}=\left\{W_{j},\left|W_{j}\right|=M_{R}\right\}$ be a set of windows generated using Relief rankings and $\mathcal{C}_{\psi}=\left\{\psi_{j}\right\}$ a set of operators such that $\psi_{j}$ was trained using $W_{j}$. We then compute a training set of second level patterns $z_{i} \in\{0,1\}^{|\mathcal{C}|}$. Each operator $\psi_{j}$ is ranked according to Mutual Information $I(z, y)$ [CT12] $\left(\mathrm{Eq} 5.10\right.$ between feature $z_{\bullet j}$ and the target variable $y$.

$$
I(z, y)=H(y)-H(y \mid z)
$$

If $I(z, y)$ is large then there is little uncertainty in the conditional distribution $y \mid z$. If $I(z, y)$ is small then conditioning $y$ on $z$ does not reduce the uncertainty significantly when compared to observing only $y$. Thus, the Mutual Information between variable $z_{\bullet} j$ and the output $y$ can be used to rank operators $\psi_{j}$.

The final step is to determine how many operators should be combined. Recall from Eq. 3.7 that the optimal operator can be fully determined if all possible window patterns are observed. In the case of two-level operators, a second-level operator $\psi^{(2)}$ is fully determined if all second-level patterns are observed. This is less likely to happen if $\psi^{(2)}$ combines a large number of operators. Therefore, the ratio of observed patterns can be used to control 
the number of operators combined.

Given a set of operators $\mathcal{C}_{\psi}^{o}=\left\{\psi_{j}\right\}$ ordered according to their MI, we define $r_{j}=\frac{T_{j}}{2^{j}}$ as the ratio between observed unique patterns $T_{j}$ and all patterns possible using the first $j$ operators in the ranking. If $r_{j}=1$ then all patterns were observed and the second-level operator is completely defined (according to Eq. 3.4). If $r_{j}<1$ then it is necessary to generalize. The number of selected operators is equal to the largest $j$ such that $r_{j}$ is larger than a desired ratio $r_{t}$.

The complete FS algorithm can be summarized in the following steps. The parameters of the algorithm are the number of candidate windows $m$, the size of the candidate windows $M_{R}$ and the desired generalization ratio $r_{t}$. FS requires the use of two training sets $T_{1}$ and $T_{2}$.

Data: Training sets $T_{1}$ and $T_{2}$, Domain window $D \subseteq \mathcal{Z}$, Number of candidates $m$, Windows size $m_{k}, r_{t}>0$

Result: Set of operators $F=\left\{\psi_{j}\right\}_{j=1}^{j^{*}}$

Generate $m$ Relief rankings from $T_{1}$;

Build $W_{j}=\left\{\right.$ top $m_{k}$ points $\}, j=1, \ldots, m$

Train $\left\{\psi_{j}\right\}_{j=1}^{m}$ using windows $\left\{W_{j}\right\}_{j=1}^{m}$ and $T_{1}$;

Compute $\left\{\left(z_{i}, y_{i}\right)\right\}$ using $\left\{\psi_{j}\right\}_{j=1}^{m}$ on training set $T_{2}$

Rank operators $\left\{\psi_{j}\right\}_{j=1}^{m}$ according to the MI between $z_{\bullet j}$ and $y$;

$j^{*}=\operatorname{argmax}_{j}\left\{r_{j}>r_{t}\right\}$

$F=\left\{\psi_{j}\right\}_{j=1}^{j^{*}}$

Algorithm 2: FS method

\subsection{A regularization based approach using linear models}

As with traditional feature selection methods in ML, the methods presented in the previous section first select operators (features) and then build the combination operator $\psi^{(2)}$. The model selected for $\psi^{(2)}$ does not influence which features are selected and different models may have different optimal feature subsets with respect to the same training set. Also, feature selection techniques might not perform well when selecting between highly-correlated features GE03. A better approach would be to do model training and feature selection at the same time. Many linear models generate sparse solutions by adding a $L_{1}$ regularization term (LASSO, L1SVM, Elastic Net, etc). The number of non-zero weights is controlled by the regularization parameter $\lambda$. Large $\lambda$ values tend to produce models that rely on smaller feature subsets. Thus, we can control $\operatorname{card}\left(\psi^{(2)}\right)$ by adjusting $\lambda$. We can also exploit sparsity to optimize over the full set $\mathcal{C}_{\psi}$. 
The use of linear models for $\psi^{(2)}$ should not impose severe constraints on its complexity. Since the second-level patterns are already non-linear transformations of the input, the resulting model is also non-linear. In terms of Image Processing, a linear $\psi^{(2)}$ computes the weighted average of several images to produce the final output.

Typical linear models are characterized by a decision function of the form $g(x)=\operatorname{sign}\left(w^{T} z(x)\right)$, where $w, z(x) \in \mathbb{R}^{\left|\mathcal{C}_{\psi}\right|}$. Since we are expecting outputs in $\{0,1\}$, we define our linear models as $\psi^{(2)}(x ; w, z)=\frac{\operatorname{sign}\left(w^{T} z(x)\right)+1}{2}$. As $w$ is a very sparse vector, we do not need to compute $z(x)_{j}$ if $w_{j}=0$. Therefore, we only need to maintain in memory the operators corresponding to the nonzero elements of $w$.

In this work we adopt the logistic loss function [HTF09] in Eq. 5.1 due to the possibility of extending it to non-binary outputs. As explained before, we replace the $\operatorname{card}\left(\psi^{(2)}\right)$ constraint with a $L_{1}$ regularization term, resulting in Eq. 5.11.

$$
\min _{w} c(w)=-\sum_{i}^{N} y_{i} w^{T} z_{i}-\log \left(1+e^{w^{T} z_{i}}\right)+\lambda \sum_{j=1}^{2^{M}}\left|w_{j}\right|
$$

To solve Eq. 5.11 we need first to compute the second-level patterns $z_{i}$, which requires training all $2^{M}$ operators in $\mathcal{C}_{\psi}$ and then applying them to each $x_{i}$. This is prohibitively expensive in both memory and time. Although we could restrict $\mathcal{C}_{\psi}$ like earlier works, we instead adopt an iterative optimization method [FYR11 to minimize Eq. 5.11.

First, we observe that the cost $c(w)$ is non-differentiable at point $w$ if $w_{j}=0$. Fortunately, $c(w)$ belongs to the class of convex functions. A function $c$, with domain $\operatorname{dom} c$, is convex if

$$
c(\alpha a+(1-\alpha) b) \leq \alpha c(a)+(1-\alpha) c(b)
$$

for all $a, b \in \operatorname{dom} c$ and $\alpha \in[0,1]$. A subgradient $g$ of a (not necessarily convex) function $c$ at point $w$ is an underestimator of $c$ in every point of its domain.

$$
c\left(w_{0}\right) \geq c(w)+g^{T}\left(w_{0}-w\right), \forall w_{0} \in \mathbf{d o m} c
$$

The set of subgradients of $c$ at $w$ is denoted as $\partial c(w)$. If $c(w)$ is convex then at least one subgradient exists for every $w \in \operatorname{dom} c$. If $c$ is differentiable at $w$ then $\partial c(w)=\{\nabla c(w)\}$. Notice, however, that $\partial c(w)$ is defined even when $c$ is non-differentiable at $w$.

If $0 \in \partial c\left(w^{*}\right)$ for some point $w^{*}$ then $c\left(w_{0}\right) \geq c\left(w^{*}\right)+0^{T}\left(w_{0}-w\right)$, which implies that $w^{*}$ is a global minimum of $c$. Thus, we need only to check first order optimality conditions of Eq. 5.11 in order to find its solution. 
Also, subgradients may provide a descent direction for points where $c$ is not differentiable.

The gradient of the logistic loss (first part of $c(w)$ ) is given below. We adopt $p_{i}=\frac{e^{w^{T} z_{i}}}{1+e^{w^{T} z_{i}}}=P\left(y=1 \mid z=z_{i}\right)$ to shorten the notation.

$$
\nabla_{w_{j}}\left(-\sum_{i=1}^{N} y_{i} w^{T} z_{i}-\log \left(1+e^{w^{T} z_{i}}\right)\right)=-\sum_{i=1}^{N}\left(y_{i}-p_{i}\right) z_{i, j}
$$

The subgradient of $g_{j}$ of the absolute term $\left|w_{j}\right|$ when $w_{j}=0$ is

$$
\begin{array}{r}
|v| \geq 0+g_{j}(v-0) \\
|v| \geq g_{j} v \\
-v \leq g_{j} v \leq v \\
-1 \leq g_{j} \leq v
\end{array}
$$

Thus, the subgradient $g$ of $\lambda \sum_{j=1}^{2^{M}}\left|w_{j}\right|$ is defined below.

$$
g_{j}=\left\{\begin{array}{l}
\lambda \quad w_{j}>0 \\
-\lambda \quad w_{j}<0 \\
{[-\lambda, \lambda] \quad w_{j}=0}
\end{array}\right.
$$

Let $w^{*}$ be a solution to Eq. 5.11. Since $0 \in \partial c\left(w^{*}\right)$, for each $w_{j}^{*}$ the following conditions hold. If a solution violates these conditions for some $j$ then it is not optimal.

$$
\begin{aligned}
& -\sum_{i=1}^{N} z_{i, j}\left(y_{i}-p_{i}\right)+\lambda=0 \quad \text { if } w_{j}>0 \\
& -\sum_{i=1}^{N} z_{i, j}\left(y_{i}-p_{i}\right)-\lambda=0 \quad \text { if } w_{j}<0 \\
& -\lambda \leq-\sum_{i=1}^{N} z_{i, j}\left(y_{i}-p_{i}\right) \leq \lambda \quad \text { if } w_{j}=0
\end{aligned}
$$

Given a set of indexes $\mathcal{A}$, we can obtain a solution $W$ that satisfies Eqs. $5.17 \mathrm{a}$ and $5.17 \mathrm{~b}$ by solving Eq. 5.11 restricted only to the variables in $\mathcal{A}$. We set $w_{j}=0$ if $j \notin \mathcal{A}$. As long as $\mathcal{A}$ is sufficiently small, we can solve the restricted problem directly. The set $\mathcal{A}$ is called the active set of $w$ and is formally defined as

$$
\mathcal{A}(w)=\left\{j \mid w_{j} \neq 0\right\} .
$$

Suppose that a solution $w$ is obtained by the procedure explained above and thus, satisfies optimality conditions for all $j \in \mathcal{A}(w)$. The only way to 
decrease the cost $c(w)$ is to find a $j^{\prime} \notin \mathcal{A}(w)$ that violates Eq. $5.17 \mathrm{c}$. If there exists such $j^{\prime}$ we compute a new $w^{\prime}$ using the procedure above with the set $\mathcal{A}(w) \cup\left\{j^{\prime}\right\}$. Clearly, $c(w)>c\left(w^{\prime}\right)$. We can then continue the optimization starting from $w^{\prime}$ instead of $w$. If we find a solution $w^{*}$ such that there is no $j^{\prime} \notin \mathcal{A}$ that violates Eq. $5.17 \mathrm{c}$ then $w^{*}$ is a global minimizer of Eq. 5.11.

This induces an active set approach to solve Eq. 5.11. At each iteration we sample an operator $\psi_{j}$ from $\mathcal{C}_{\psi}$, train it in training set $T_{1}$, compute $Z_{\bullet} j$ by applying it to training set $T_{2}$ and check whether it violates Eq. $5.17 \mathrm{c}$. In the positive case $j$ is included in $\mathcal{A}$ and a new solution $w^{\prime}$ is computed. Otherwise $\psi_{j}$ and $Z_{\bullet j}$ are discarded. We call this procedure NILC, a shorthand for Near Infinite Linear Combination. The algorithm is shown in Algorithm 3 .

Data: Training sets $T_{1}$ and $T_{2}$, Domain window $D \subseteq \mathcal{Z}$, Number of iterations niter, $\lambda>0$

Result: Weights $\hat{w}$

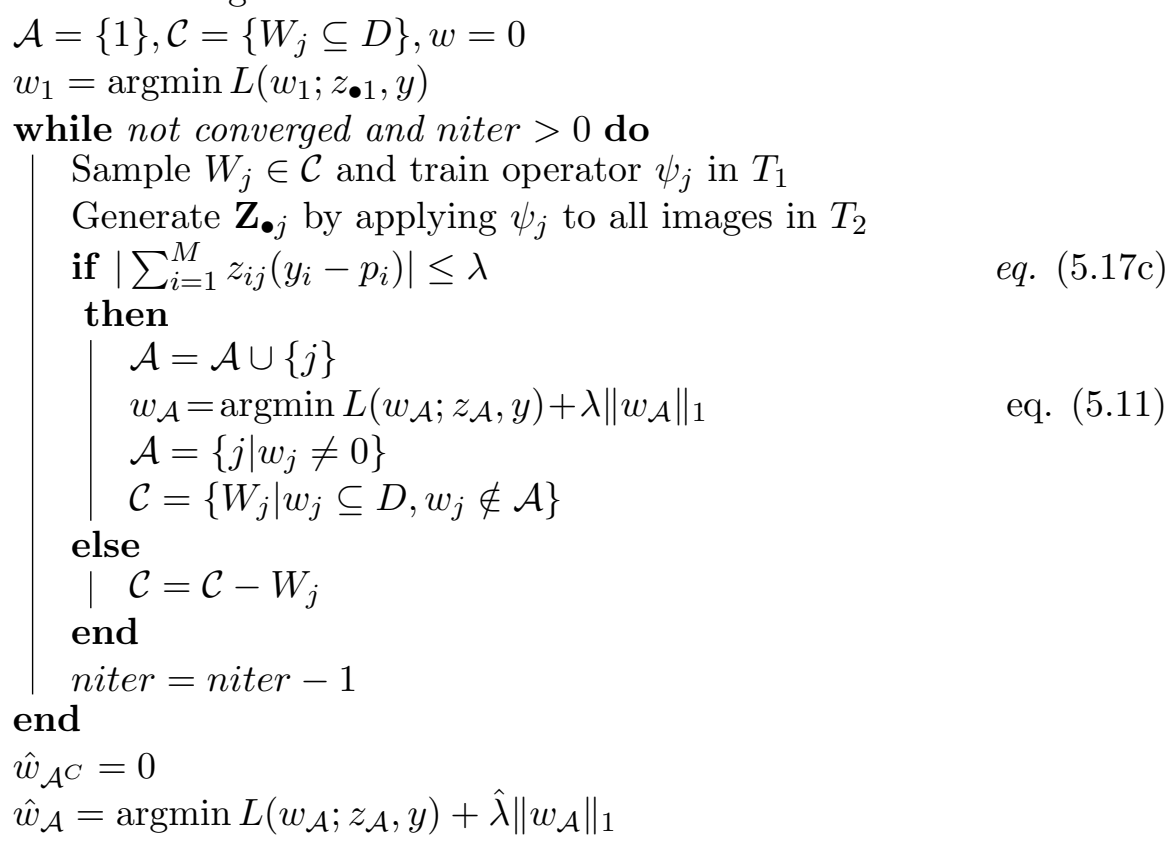

Algorithm 3: Near Infinite Linear Combination

For convenience, we set the output of the operator with the empty window, $\psi_{1}(x)$, to 1 so that the linear combination includes a bias term without explicitly adding it to the problem formulation.

Differently from previous methods, NILC can be used to improve upon an already trained operator and is guaranteed to generate a solution that is at least as good as the current one. If we acquire more data or need to adapt to different imaging conditions, we just need to run more iterations using the new data. This is valuable, for instance, in the Staff Removal problem (described in Chapter 7), where images may be produced under 
many different digitalization conditions.

NILC has several other advantages when compared to earlier methods for two-level optimization. First, it does not impose any restriction on the size of $\mathcal{C}_{\psi}$, making it possible to consider all subwindows of $D$ in the combination. Second, it defines an optimization problem in the second level pattern space and has a notion of the optimal two-level operator for a given domain window $D$. Third, it saves resources by storing only the columns of $\mathcal{Z}$ (and operators $\psi_{j}$ ) whose corresponding variables are in the active set $\mathcal{A}$. It also trains operators $\psi_{j}$ on demand and discard them as soon as they leave the active set.

Determining $\lambda$ is the main weakness of NILC. This hyperparameter must be adjusted manually and there is no automatic way to associate a certain $\lambda$ with the number of combined operators. Adequate $\lambda$ is chosen by gridsearch.

In practice we do not consider all $W \subseteq D$. Instead, we use all subwindows of a certain size $M_{W}$ (usually $M_{W}=40$ provides a good balance between generalization performance and window size). We also set $\lambda$ so as to generate combinations of at most 10-15 operators. We find that using bigger combinations do not result in significant gains in accuracy but makes the resulting two-level operator much slower. Also, we consider that NILC has converged when no progress is made in a validation set after 20 iterations.

It is also possible to adapt NILC to use other loss functions as long as Eq. 5.11 remains convex. SVMs may be used, for instance, when dealing with binary outputs. It is also possible to change the way $\mathcal{C}_{\psi}$ is built. Instead of combining operators trained with different subwindows one could, for instance, combine operators trained with different training subsamples (as in Bagging) or using different hyperparameters. We explore this in Chapter 6.3. where we use NILC to combine operators trained using KA. 


\section{Chapter 6}

\section{Experiments}

The methods presented in this work require the determination of some hyperparameters to achieve good performance. In this chapter we explore the effect of these hyperparameters on the behavior of both methods and determine good hyperparameters to be used in Chapter 7 .

Recall that, given a kernel $k$, KA computes a feature map $\phi$ that approximates kernel $k$ from a subsample of size $n_{A}$ from the training set. Then it selects another subset of the training set to train a SVM classifier using the training examples transformed by the estimated $\phi$. The hyperparameters of the algorithm are the degree $d$ of the polynomial kernel (for binary inputs), the bandwidth $\gamma$ of the Gaussian kernel (for gray-level inputs), the size $n_{A}$ of the approximation set and the number of examples to use in the final training. We evaluate the effect of these parameters in Sections 6.1 and 6.2

Recall that, given a domain window $D$, NILC computes a linear decision envolving operators trained with all subwindows $W_{j} \subset D$ of $D$. It solves a highly regularized minimization problem to determine the weights $w_{j}$ of each operator. The regularization constant $\lambda$ is related to the number of operators selected. Large $\lambda$ induces very few $w_{j} \neq 0$. We show in Section 6.3 that for a fixed $\lambda$ the number of operators selected vary in a certain range, effectively controlling the number of operators combined. We also explore combinations of KA-based operators.

We use the datasets presented in Table 6.1 in all the following experiments. Each dataset is divided in two disjoint image sets. The first one, $T_{1}$, is always used as training set. The second one is split in two parts: $T_{2}$, used as training set for two-level operators, and $V$, used as validation set.

Both jung and veja were used in many earlier works [Hir09, DH15, dSdS10 and correspond to document processing techniques. staffs-small involves the removal of staff lines from music scores. drive10 contains images for the segmentation of retinal blood vessels. Both are explained in more detail in Chapter 7. The first three datasets (veja, jung and staffs-small) have binary inputs, while drive10 has gray-level input images. 


\begin{tabular}{|l||ccc|}
\hline Name & Window size & Training size $|T|$ & Validation size $|V|$ \\
\hline jung9x7 & $9 \times 7$ & 114108 & 79337 \\
jung11x9 & $11 \times 9$ & 114023 & 79296 \\
veja9x7 & $9 \times 7$ & 669733 & 379807 \\
veja11x11 & $11 \times 11$ & 668457 & 378762 \\
staffs-small & $11 \times 11$ & 11637686 & 524239 \\
drive10 & $11 \times 11$ & 2271374 & 226968 \\
\hline
\end{tabular}

Table 6.1: Summary of datasets used in the experiments.

\subsection{Kernel hyperparameter determination for Nyström approximations in KA}

The KA method uses polynomial kernels for problems with binary input (datasets jung9x7, jung11x9, veja9x 7 , veja11x11 and staffs-small) and RBF kernels for problems with gray-level inputs (drive10). In this chapter we determine adequate hyperparameters for each dataset.

For the binary input problems we have tested kernels using polynomials of degree $d \in\{3,5,7,9\}$ using approximation samples $N_{a} \in$ $\{100,500,1000,2000\}$ examples. We train a SVM with a sample of 100,000 observations from $T_{1}$ and compute the error on the validation set $V$.

We have tested RBF kernels with bandwidth $\gamma \in$ $\{0.1,0.01,0.001,0.0001\}$ and approximation sets of size $N_{a} \in$ $\{100,500,1000,2000\}$ for the drive10 dataset. We follow the same training procedure adopted in the binary case.

Results for all six datasets are shown in Figure 6.1. The selected parameters for each dataset are shown in Table 6.2. In the binary cases the parameters $d=3, N_{a}=2000$ were selected for datasets jung $9 x 7$, jung11x9, veja11x11 and staffs-small. We use $d=5, N_{a}=2000$ in veja9x\%. Although in some cases $d=5, N_{a}=2000$ achieved very similar error rates, we opted for $d=3$ to reduce model complexity. We have observed higher variance when approximating polynomials with degrees 7 and 9, as shown in Figure 6.1e. In the gray-level case $\gamma=0.01, N_{a}=2000$ was the best parameter combination. Correctly tuning $\gamma$ is a necessary step to use KA in this context, as the difference between the smallest error and the largest is close to $1 \%$. When using the selected parameters, increasing $N_{a}$ decreased validation error.

\subsection{Random behavior of the KA algorithm}

For a given set of kernel hyperparameters, the KA algorithm has two sources of randomness that may affect its performance. The first one is the computation of the Nyström approximation $\phi$, which depends on the approximation 


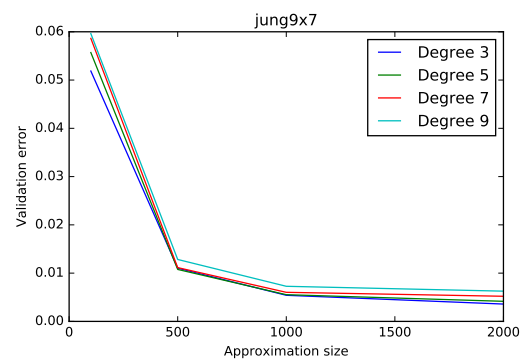

(a)

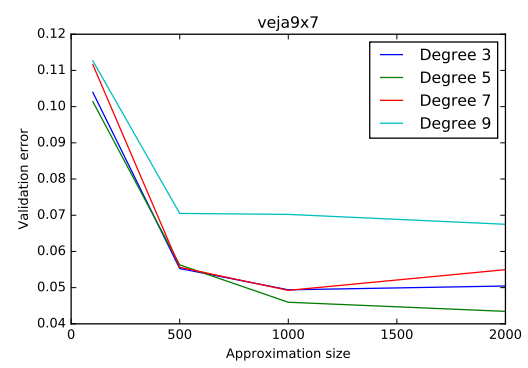

(c)

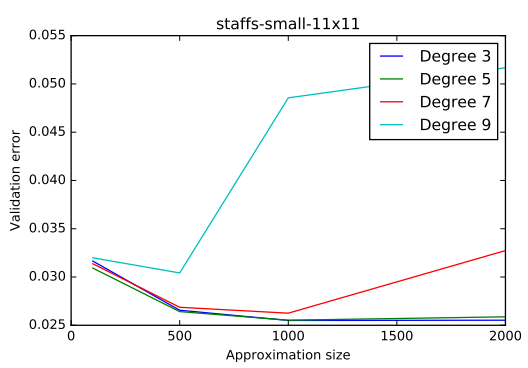

(e)

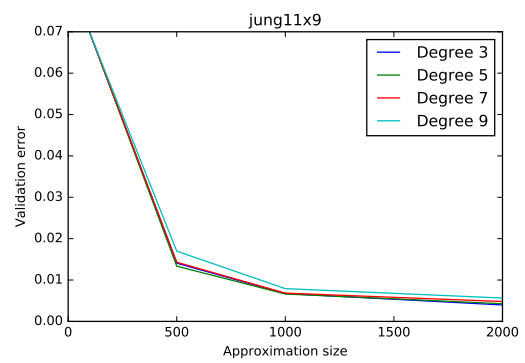

(b)

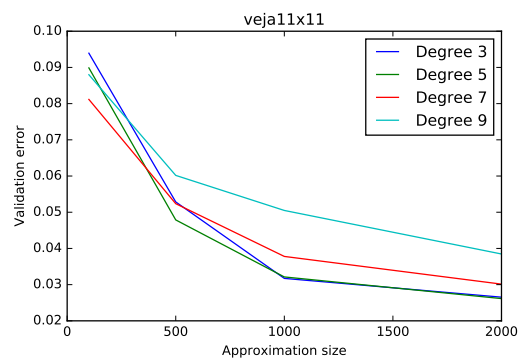

(d)

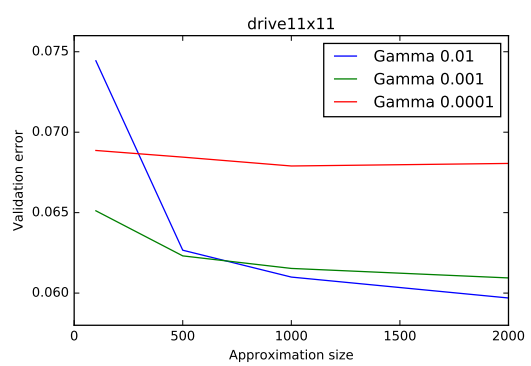

(f)

Figure 6.1: Plots (a) to (e) show the validation performance of a polynomial kernel with increasing degree for problems with binary input. Plot (f) shows the validation performance of a Gaussian kernel with different bandwidths for a problem with gray-level inputs. 


\begin{tabular}{|l||cc|}
\hline Dataset & $N_{a}$ & Parameter \\
\hline \hline jung9x' & 2000 & $d=3$ \\
jung11x9 & 2000 & $d=3$ \\
veja9x' & 2000 & $d=5$ \\
veja11x11 & 2000 & $d=3$ \\
staffs-small & 2000 & $d=3$ \\
\hline drive10 & 2000 & $\gamma=0.01$ \\
\hline
\end{tabular}

Table 6.2: Selected hyperparameters for KA based on the validation performance. $N_{a}$ is the size of the approximation set, $d$ is the degree of the polynomial kernel and $\gamma$ is the bandwidth of the Gaussian kernel.

set $\left(x_{i}^{\prime}, y_{i}^{\prime}\right), i=1, \ldots, m$ chosen to compute it. Good approximations are essential to obtain a solution close to the optimum of the full kernel SVM (Eq 2.19). The algorithm presented in Chapter 4 uses random sampling from the training data to select the approximation examples.

The second source of randomness is the choice of examples to train the SVM. Both size and choice of the subset of training examples used can impact the performance of the resulting operator. We have designed two experiments to measure the effect of these choices in the outcome of KA.

Experiment 1: We fix the sample used to train the SVM and analyze the variance induced by the Nyström approximation. First, we select 30 different approximation sets of $m=2000$ examples. Then we compute approximations $\phi_{i}, i=1, \ldots, 30$, train a KA operator for each $\phi_{i}, i=1, \ldots, 30$ and measure the errors in the validation set. The kernel hyperparameters for each dataset were the ones determined in the previous section (Table 6.2). We use the same set of examples to train the SVMs of all operators.

The results of Experiment 1 are shown in Table 6.3. We have computed the mean and standard deviation of all executions and compared them with the validation performance of a Decision Tree trained with all data. In all cases the mean KA validation error was significantly smaller than the DT's. The standard deviation was also small, indicating that the choice of approximation set does not influence the result heavily.

Experiment 2: We select a single approximation set and compute $\phi$ using the hyperparameters from Experiment 1 (Table 6.2). Then, for every $n$ from 20000 to 200000 in increments of 20000, we select 30 different subsets of $n$ training samples to train 30 SVMs and measure their errors in the validation data.

The results of Experiment 2 are shown in Figure 6.2. In all cases the mean error decreases when more data is used to train the SVMs. The standard deviations also decrease as more data is used.

In both experiments the mean performance of the KA operators was, at the worst case, equivalent to a Decision Tree. The standard deviations 


\begin{tabular}{|l|cc||c|}
\hline Dataset & Mean & Std & DT error \\
\hline jung9x7 & 0.0042 & $11 \times 10^{-5}$ & 0.0132 \\
jung11x9 & 0.0043 & $16 \times 10^{-5}$ & 0.0139 \\
veja9x7 & 0.046 & $8 \times 10^{-4}$ & 0.039 \\
veja11x11 & 0.026 & $8 \times 10^{-4}$ & 0.035 \\
staffs-small & 0.022 & $8 \times 10^{-5}$ & 0.025 \\
drive10 & 0.061 & $1 \times 10^{-4}$ & 0.113 \\
\hline
\end{tabular}

Table 6.3: Mean validation performance of KA using 30 different approximations trained using a fixed training set. The last column shows the error of Decision Tree (DT) trained with the same training set.
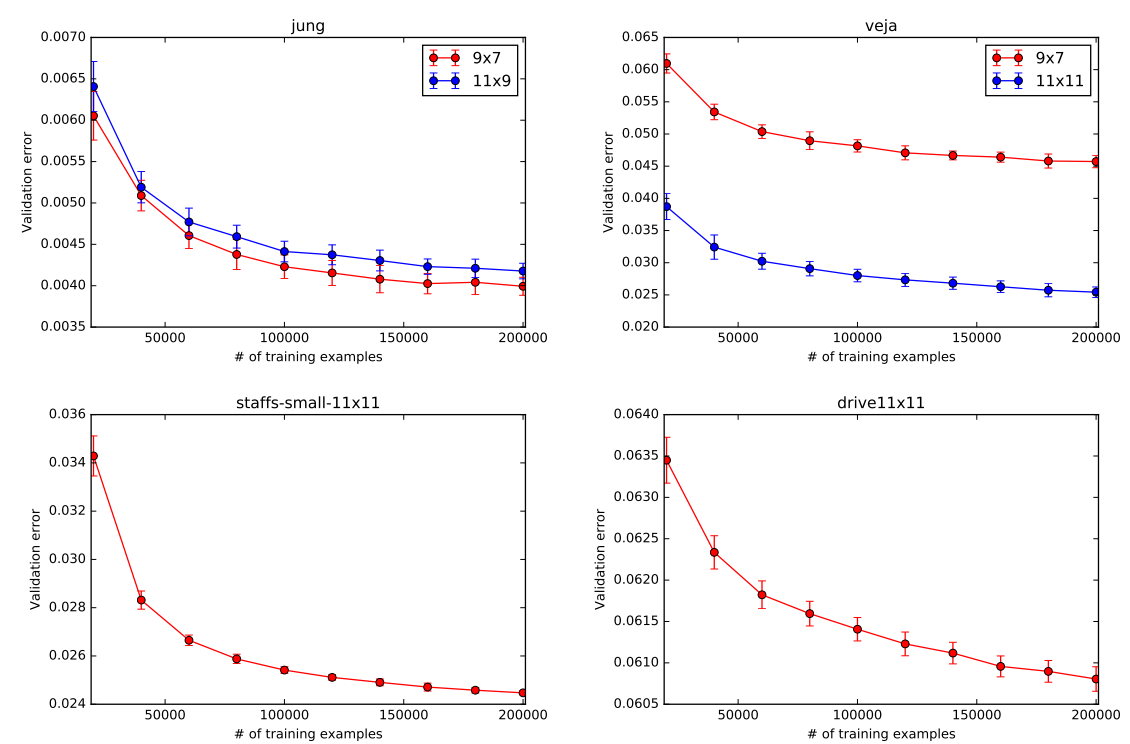

Figure 6.2: Mean validation error from SVMs trained with progressively more data and a fixed approximation set. Mean and standard deviation of 30 executions.

of the validation performances were also small, as seen in Table 6.3 and Figure 6.2. The effect of varying the hyperparameters, on the other hand, was much larger (as shown in Figure 6.1). The best hyperparameter set had significantly smaller validation performance than the others. Thus, as expected, the hyperparameters of the kernels impact the performance more significantly than the random choices made by KA. 


\subsection{Determining $\lambda$ for NILC}

The $\lambda$ parameter controls the number of combined operators in NILC. Its value has a strong relation with the logistic error term of Eq. 5.11. The larger the logistic error the smaller $\lambda$ must be in order to add more operators and decrease the cost.

As reported in previous works [MHH14b, MHH16, MSHC], combinations with more than approximately 15 operators do not present significant gains in performance when compared with smaller combinations. Thus, we manually determined $\lambda$ by trial and error to select around 10-15 operators for each dataset.

In this chapter we compare combinations using Decision Trees and KA in the first-level. We refer to operators trained with each classifier as, respectively, NILC-DT and NILC-KA. The $\lambda$ values used in NILC-DT and NILC-KA are shown, respectively, in Table 6.4.

\begin{tabular}{|l||cc|}
\hline Name & $\lambda_{D T}$ & $\lambda_{K A}$ \\
\hline jung9x7 & 4.2 & 6 \\
jung11x9 & 3 & 2.5 \\
veja9x7 & 20 & 10 \\
veja11x11 & 23 & 10 \\
staffs-small & 60 & 70 \\
drive10 & 0.5 & 5 \\
\hline
\end{tabular}

Table 6.4: $\lambda$ values for NILC-DT and NILC-KA.

We show in Figure 6.3 the progress of NILC-DT in datasets jung11x9, veja11x11, staffs-small and drive10. We stop NILC when 20 iterations pass without improvement in training error and keep the best combination found. As expected, after some iterations the number of operators combined stays within a certain range. Also, although the cost does not correspond exactly to error, decreasing the cost provokes a decreasing trend in the error.

The dataset drive10 produced the most impressive results with NILCDT. The error decreases from approximately $12 \%$ to about $6.4 \%$, similar to operators using $\mathrm{KA}$ in the previous chapters. In all other datasets the performance of the two-level operators was much superior to the first-level ones.

We show in Figure 6.4 the validation error on each iteration for NILCKA. Instead of sampling different subwindows, in each iteration we sample a different subset of the data to build the Nyström approximation and another subset of size 100, 000 to train the SVM. Again, we stop after no progress is obtained in the training error for 20 iterations.

The decrease in training error is much smaller in NILC-KA. This can be attributed to the small variance of the SVMs (shown in Table 6.3 and 


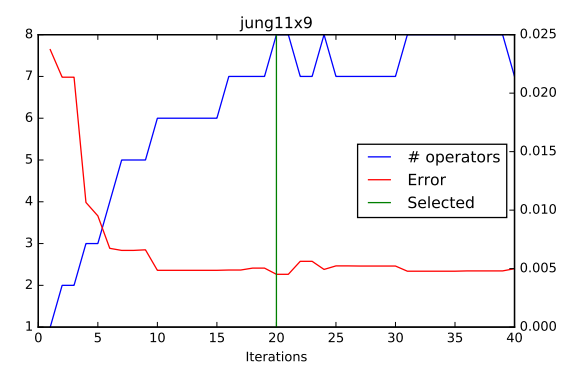

(a)

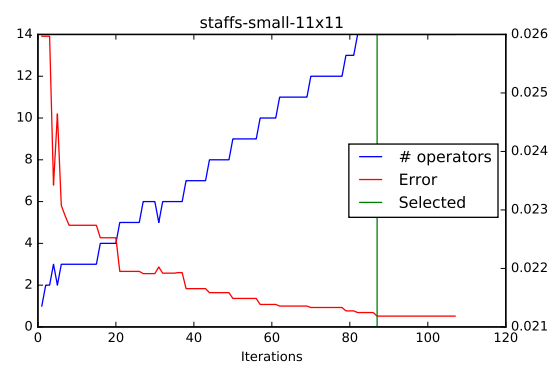

(c)

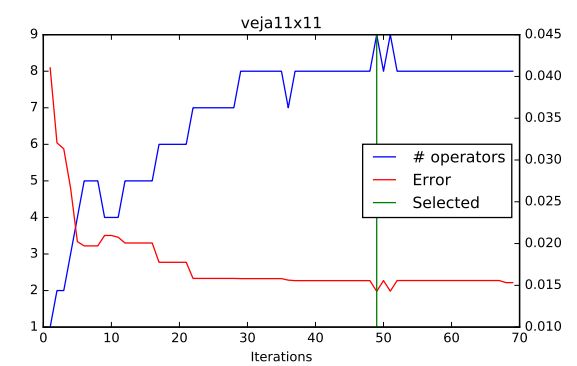

(b)

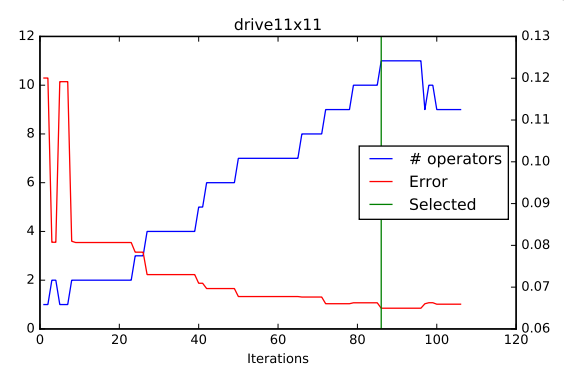

(d)

Figure 6.3: Training error (left axis) and number of selected operators (right axis) in each iteration of NILC-DT. Training error decreases significantly in all datasets.

Figure 6.2), as the most operators agree and not much can be gained from aggregating their outputs. There is definitely some improvement in the harder tasks (staffs-small and drive10), although the results shown in Figure 6.2 might indicate that similar improvements could be achieved by training an SVM using more data.

The validation performance of resulting operators from both NILC-DT and NILC-KA is shown in Table 6.5. The performance of both NILC-DT and NILC-KA were similar, although NILC-DT improved results dramatically when compared to its first-level operators. NILC-KA, on the other hand, only showed small improvements when compared to the first-level KA operators.

\subsection{Generalization performance}

In this section we analyze test performance for KA, NILC-DT and NILC-KA for datasets jung9x 7 , jung11x9, veja9x 7 and veja11x11. The performance on datasets staffs-small and drive10 is explored in more detail in Chapter 7 .

We show in Table 6.6 the test performance of the studied methods and compare it to earlier methods for two-level optimization in two test sets $T_{3}$ 


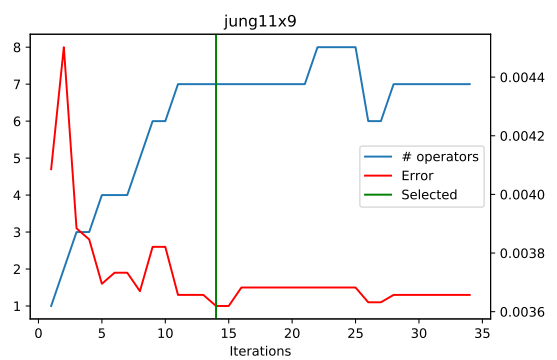

(a)

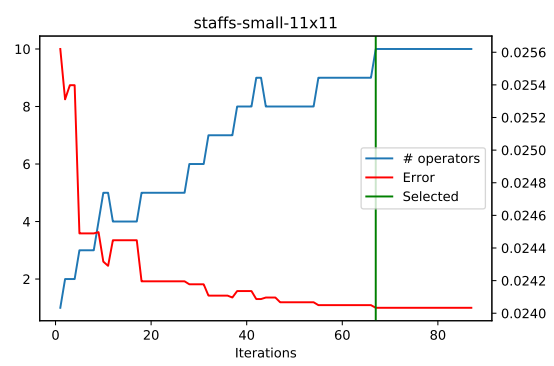

(c)

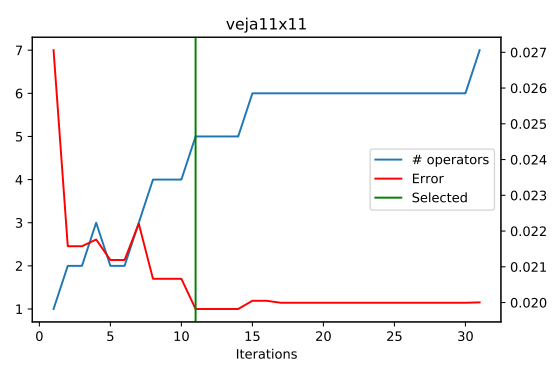

(b)

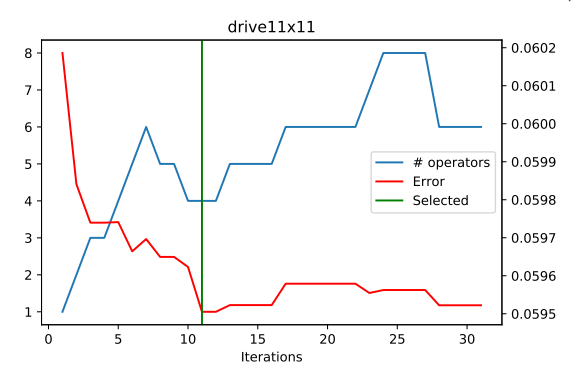

(d)

Figure 6.4: Training error (left axis) and number of selected operators (right axis) in each iteration of NILC-KA. Training error does not decrease significantly.

and $T_{4}$.

The performance of KA, NILC-DT and NILC-KA is superior to WER, IT and the manual approach used in [Hir09]. KA obtained results very close to the combination NILC-KA, indicating that combining SVMs does not result in significantly better performance for these problems. This has also been observed during validation (Table 6.5 and Figure 6.4). NILC-DT had the smaller errors in the veja datasets, while NILC-KA was better in jung. 


\begin{tabular}{|l||ccc|}
\hline Dataset & NILC-DT & NILC-KA & KA \\
\hline jung9x 7 & $0.004 / 8$ & $0.003 / 5$ & 0.004 \\
jung11x9 & $0.004 / 8$ & $0.003 / 7$ & 0.004 \\
veja9x7 & $0.017 / 10$ & $0.034 / 6$ & 0.046 \\
veja11x11 & $0.014 / 9$ & $0.019 / 5$ & 0.026 \\
staffs-small & $0.021 / 14$ & $0.023 / 10$ & 0.022 \\
drive10 & $0.062 / 13$ & $0.058 / 4$ & 0.061 \\
\hline
\end{tabular}

Table 6.5: Validation performance and number of operators for NILC-DT and NILC-KA. We show in the last column the mean performance of KA operators from Table 6.3. NILC-KA does not improve significantly over single KA operators.

\begin{tabular}{|c|c|c|c|c|c|}
\hline \multirow{2}{*}{ Domain } & \multirow{2}{*}{ Method } & \multicolumn{2}{|c|}{ jung } & \multicolumn{2}{c|}{ veja } \\
\cline { 3 - 6 } & & $T_{3}$ & $T_{4}$ & $T_{3}$ & $T_{4}$ \\
\hline \multirow{5}{*}{$9 \times 7$} & NILC-DT & 0.0045 & 0.0045 & $\mathbf{0 . 0 3 4}$ & $\mathbf{0 . 0 2 0}$ \\
& NILC-KA & $\mathbf{0 . 0 0 3 5}$ & $\mathbf{0 . 0 0 4 1}$ & 0.037 & 0.026 \\
& KA & 0.0036 & $\mathbf{0 . 0 0 4 1}$ & 0.036 & 0.029 \\
& IT & 0.007 & - & - & - \\
& WER & 0.0041 & 0.0046 & 0.037 & 0.022 \\
& Manual & 0.006 & 0.005 & 0.046 & 0.026 \\
\hline \hline \multirow{5}{*}{$11 \times 9$} & NILC-DT & 0.0044 & 0.0044 & - & - \\
& NILC-KA & $\mathbf{0 . 0 0 3 1}$ & 0.0037 & - & - \\
& KA & 0.0035 & 0.0043 & - & - \\
& Manual & 0.004 & - & - & - \\
\hline \hline \multirow{5}{*}{$11 \times 11$} & NILC-DT & - & - & 0.026 & $\mathbf{0 . 0 1 5}$ \\
& NILC-KA & - & - & 0.024 & $\mathbf{0 . 0 1 5}$ \\
& KA & - & - & $\mathbf{0 . 0 2 2}$ & 0.016 \\
& IT & - & - & 0.031 & - \\
& Manual & - & - & 0.031 & - \\
\hline
\end{tabular}

Table 6.6: Comparative test errors for datasets jung and veja. A "-" indicates that the authors of the method did not test a certain combination of window size and training set. 


\section{Chapter 7}

\section{Applications}

In this chapter we describe two application domains where $W$-operator learning achieves results competitive with classical algorithms in the literature. The first application, called Staff Removal, is a common preprocessing in Optical Music Recognition. The second application is the segmentation of blood vessels in retinal images. We evaluated both NILC and KA in public datasets from both domains and obtained encouraging results.

\subsection{Staff removal}

Optical Music Recognition (OMR) systems aim to recognize digitized music scores and convert them into a machine-readable form $\left[\mathrm{RFP}^{+} 12\right]$. A very common step in the pipeline of OMR systems is removing the music staves from the images. This transformation segments the written (or printed) musical symbols from the background of the page and, at the same, facilitates the recognition of the symbols $\left[\mathrm{RFP}^{+} 12\right.$. An example of the application of this processing can be seen in Figure 7.1 .

Although this problem seems simple at first, there are many common imperfections that can affect the performance of an OMR system. The scanned paged may have folds or dents, specially when dealing with old documents. Staff lines may not be parallel to each other or they may be curved instead of completely horizontal. The contents (symbols and annotations) may be handwritten, which adds even more variability to the process. For this rea-

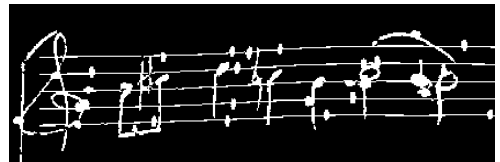

(a) Input

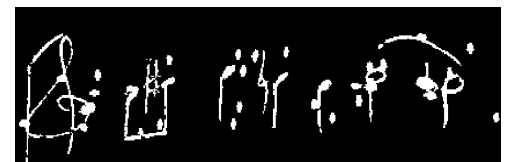

(b) Expected output

Figure 7.1: Input-output pairs for the staff removal problem. 

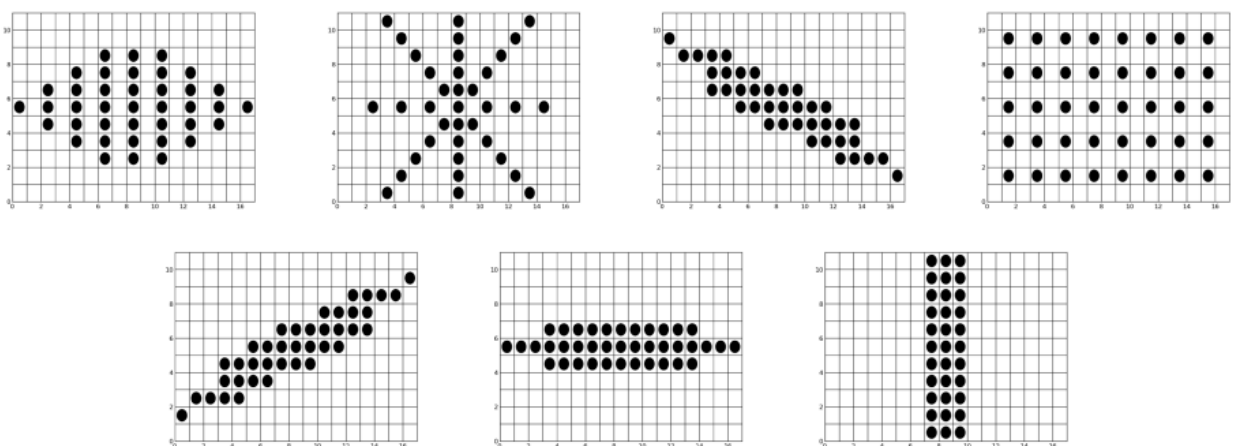

Figure 7.2: Reference windows for staff removal. Black points are used as features in training.

son several algorithms have been developed to do staff removal. Dalitz et al DDPF08] evaluated the performance of classical methods in many different deformation contexts and concluded that no tested method achieved good performance in all contexts, although all methods were very good when treating undeformed images. Thus, staff removal is a hard problem in uncontrolled conditions.

In a preliminary work $\mathrm{MHH16}$ we analyzed the robustness of learned operators using the Dalitz dataset and using a set of 7 reference windows (shown in Figure 7.2) that performed well for staff removal. These windows were manually determined by taking into account common shapes found in music scores, such as lines (horizontal, vertical and diagonal) and squares and circles. In this work they are used as benchmark for the FS algorithm presented in Section 5.2 .

The Dalitz dataset contains only 32 computer generated images. Deformed versions of this dataset are obtained by using deformation models that simulate real digitalization conditions. In previous works MHH14a, MHH16 we have observed that splitting these images in training and test sets could easily produce training tests with different characteristics from the test set. Although the generated operators were able to cope with this difference to some extent, we opted instead to use a larger dataset. The CVC-MUSCIMA dataset FDGL12 contains 1000 digitized music scores consisting of 20 music scores handwritten by 50 musicians. A variation of this dataset, which we refer to as staffs dataset, was the focus of a competition in ICDAR 2013 [VKFJ13, FVD $^{+} 13$. Images were artificially deformed to simulate conditions found in old documents, resulting in 2000 images for training and 1000 for testing. Many different methods were submitted to the competition, including Skeleton and LTC, described in [DDPF08, and LRDE [Gí4], the winner of the competition.

The competition used the following measures to compare the perfor- 
mance of the tested algorithms: Accuracy, Recall (ratio of pixels correctly classified as staff lines), Specificity (ratio of pixels correctly identified as non staff lines) and F1 (balance between Precision and Recall). Models with high Recall remove most of the staff lines successfully. Models with high Specificity do not degrade the musical symbols. Models with high F1 have a good balance between correctly removing staff lines and preserving the symbols. Ideally we would like to build models with high Recall, Specificity and F1.

\section{Analysis of the FS method}

The Relief rankings used in to generate first-level windows in the FS method can also be used to analyze discriminant shapes in a dataset. Features that induce discrimination between staff-line and non staff-line pixels should have high scores in rankings produced by Relief. Since each feature represents a point inside domain window $D$, if a set of features appears consistently at the top of the ranking then the shape formed by their corresponding points inside window $D$ is an important visual cue to determine the value of the output pixel. It may be necessary to generate many rankings in order to identify what features/points are in fact relevant and what shapes are present, as there is some variance in the computed rankings. However, as reasoned before, discriminant point/features should be present in all rankings and, thus, important shapes should be revealed by analysing many Relief rankings.

We test the use of Relief to identify shapes in the data in two scenarios. In both scenarios we generated 20 Relief rankings using a set of 200 prototypes and used the top 40 ranked points in each ranking as a window. We then count how many times each point occurred in the generated windows. In the first scenario we select a subset of 50 images to compute the rankings and select 20 prototype sets using different random seeds. In the second scenario we fix the random seed used to select the 20 prototype sets but use different disjoint subsets of 50 images to generate the rankings.

We present the results of both scenarios in Figure 7.3. The color represents the frequency that each point occurred in the generated windows. Points in dark blue did not appear in any of the generated windows, while points in dark red were present in all 20 windows. Most pixels in the horizontal center line were selected in almost all windows, while some points at the border near the center line were also considered important in a significant portion of windows. This structure coincides with the general shape of staff-lines, which are long, horizontal lines. All other points of the window do not appear in a significant portion of windows. Results are consistent in both scenarios, which indicates that Relief is able to find this structure independent of a certain combination of dataset/prototype set. The inclusion of other points in the windows helps to keep the generated windows diverse, 

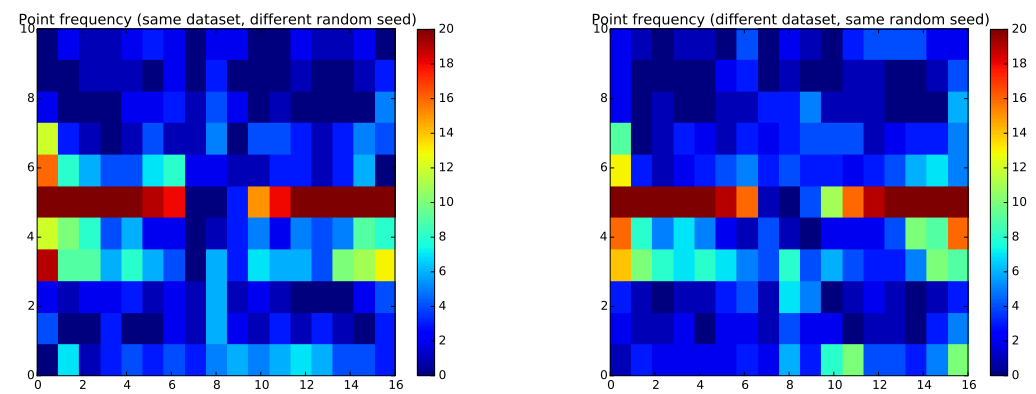

Figure 7.3: Point frequency for a set of Relief rankings computed in two different scenarios. Redder points were selected by a larger number of executions, while bluer points were less common.
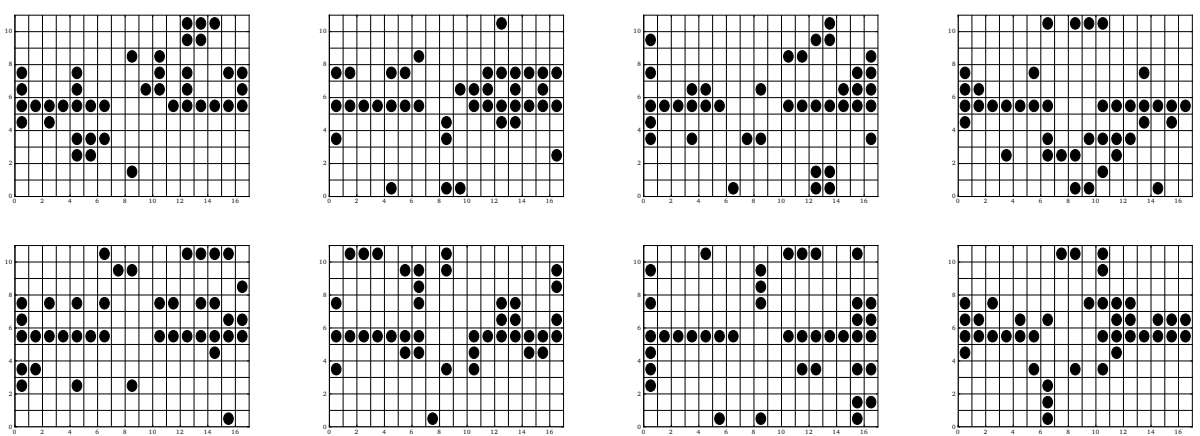

Figure 7.4: Windows found by the FS method. Black points are used as features in training.

while the horizontal center line should induce good decision functions.

\section{Performance evaluation}

We executed the FS method using a set of 50 images for the first level operators and 20 images for the second-level operators. We generated 40 candidate first-level windows and selected a ratio of 0.9 for the second-level ranking, resulting in a combination of 15 windows. Representative examples are shown in Figure 7.4. Since we consider a small set of candidate windows in FS, we use more images in the first-level training. In the case of iterative methods like NILC this cost may become prohibitive, as it may be necessary to train a large number of operators for the method to converge. As a benchmark we trained an operator using the reference windows (Figure 7.2) and the same datasets as FS.

We use the NILC-DT operator trained in Section 6.3. It was trained using a $11 \times 11$ domain window, 20 images for the first-level operators and another set of 10 images for the second-level training. Since NILC-KA and 


\begin{tabular}{|l|c|c|c|c|}
\hline Method & Acc (\%) & Rec (\%) & Spec (\%) & F1 \\
\hline LTC & 87.58 & 64.66 & $\mathbf{9 9 . 5 3}$ & 80.40 \\
Skeleton & 94.50 & 86.97 & 99.03 & 92.24 \\
LRDE & 97.03 & 94.02 & 98.84 & $\mathbf{9 5 . 9 7}$ \\
\hline \hline FS & $\mathbf{9 7 . 3 4}$ & $\mathbf{9 5 . 8 7}$ & 98.22 & 95.90 \\
Reference & 96.89 & 94.37 & 98.41 & 95.51 \\
\hline \hline NILC-DT & 96.77 & 93.85 & 98.52 & 95.62 \\
KA11 & 96.27 & 92.92 & 98.28 & 94.93 \\
KA17 & 96.52 & 93.42 & 98.39 & 95.28 \\
\hline
\end{tabular}

Table 7.1: Comparative results, accuracy (Acc), recall (Rec), specificity (Spec) and F1-measure (F1), for the staffs dataset.

NILC-DT obtained very similar results in the validation we opted for the former, as it is much faster to apply.

We trained a KA operator using two windows $(11 \times 11$ and $11 \times 17)$, a polynomial kernel with $d=3, N_{a}=2000$ and 200,000 examples. Both the components for the kernel approximation and the training examples were extracted from a set of 20 images. The results of FS,NILC-DT and KA on the test set are shown in Table 7.1.

The Skeleton, LTC and LRDE authors also published implementations of their methods. We executed each heuristic method using their default parameters and included their results in Table 7.1.

The best heuristic method in our evaluation was LRDE, followed by Skeleton and LTC. The Recall of LRDE was much larger than Skeleton and LTC, while the Specificity of all three methods was similar. This means that LRDE removed staff lines much better while still preserving the symbols.

The FS method had the best results between all learned $W$-operators, while the Reference windows placed second. However, the comparison of their results to NILC-DT and KA is not direct, as they used a much bigger number of images during training. Taking this into consideration, the results obtained by NILC-DT and KA are actually very good. NILC-DT uses less than half the number of images of FS and a smaller window, but achieves performance close to the Reference windows and is not far from FS. NILC-DT is also very close to LRDE and beats Skeleton and LTC. When comparing the other measures, NILC-DT preserves the symbol elements better than FS and Reference, but has a smaller staff detection rate.

The results of KA are even more impressive: even though it is currently limited to using only a small subset of the training set, it is only about $1 \%$ worse than FS and still better than Skeleton and LTC. Increasing window size also increased all four measures. KA differs in this point from all other learned methods. Although there seems to be a trade-off between Recall 


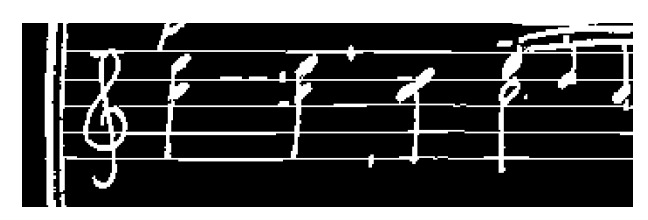

(a) Input

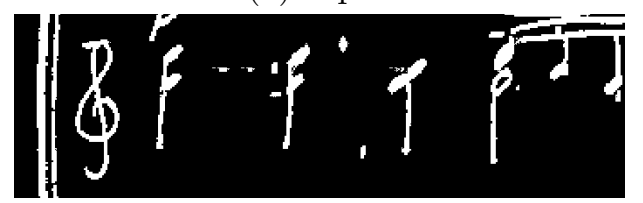

(c) FS

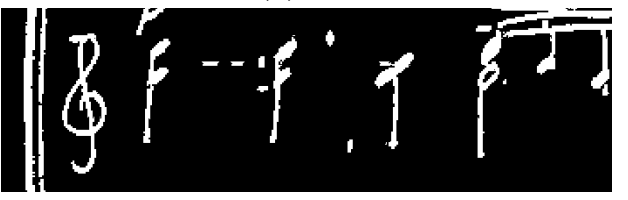

(e) NILC

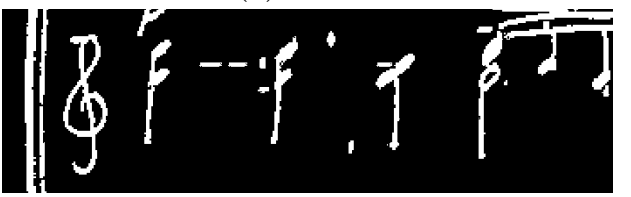

(g) KA11

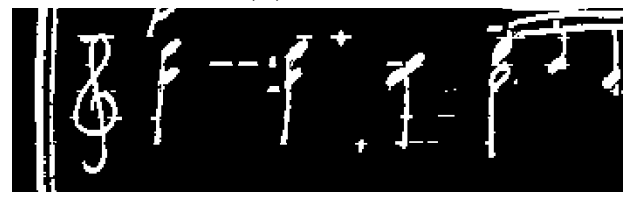

(i) $\mathrm{LTC}$

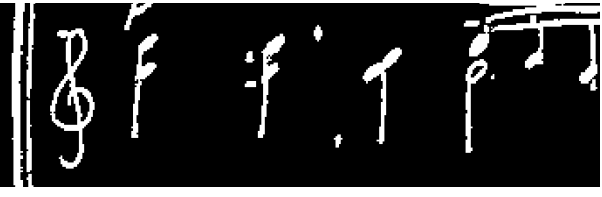

(b) Ideal

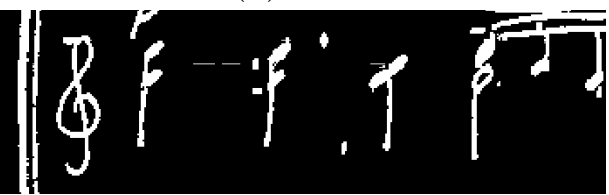

(d) LRDE

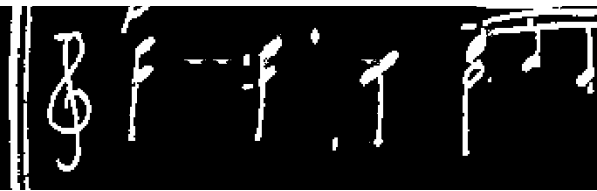

(f) Reference

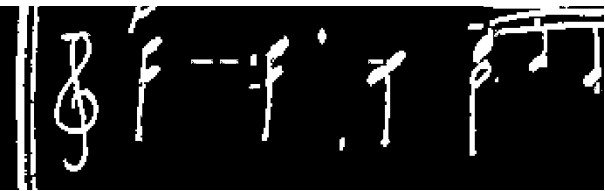

(h) KA17

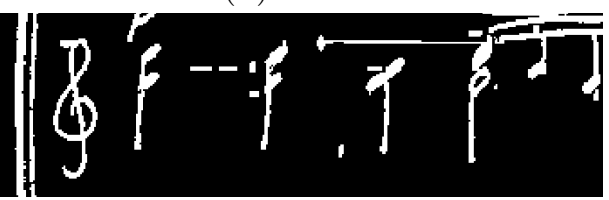

(j) SKEL

Figure 7.5: Example of the output of different methods in the staffs dataset. 
and Specificity in NILC-DT, FS and Reference, using a bigger window in KA increased both measures. The difference between Reference and KA17 is mainly due to Recall, as Specificity is about the same.

A visual comparison of all methods is shown in Figure 7.5. FS and LRDE both have obtained the best results, although LRDE is slightly better at removing staff lines. NILC had similar results to the Reference windows and KA17 was slightly better than KA11. Finally, LTC and Skeleton did not remove many staffline segments. The main difference between LRDE and FS was the type of error each method committed. LRDE removed stafflines perfectly in most cases but sometimes missed large segments. FS, on the other hand, sometimes missed small staffline segments. This happened more frequently when the stafflines changed thickness. This characteristic is shared between all learning based methods (NILC, Reference, KA11 and KA17), which differed mostly in their ability to remove stafflines.

In this section we examined the performance of learned operators applied to the staff removal problem. We conclude that $W$-operator learning is a suitable tool for this task, as it is able to produce results comparable with state-of-the-art algorithms while still being able to adapt to different digitalization conditions. In a more in-depth work [MHH16] we also show that learned operators are robust to many types of deformations commonly found in digitized music scores.

\subsection{Blood vessel segmentation in retinal images}

Many medical diagnoses can be made by measuring the characteristics of blood vessels such as vessel width and number of branches [SAN $\left.{ }^{+} 04\right]$. These can be obtained by analyzing photographs of the back of the eye (retinal images). Automatic segmentation of the blood vessels is necessary when a large number of images must be analyzed. The DRIVE dataset $\mathrm{SAN}^{+} 04$ was proposed to evaluate different methods for blood vessel segmentation in retinal images. It contains 40 images with ground truth split into 20 images for training and 20 images for testing. Each image in the test set has two segmentations: a gold standard and one made by a human observer. The latter can be used to compare automatically created segmentations and ones made manually by a trained user. An example of an input-output pair from DRIVE is shown in Figure 7.6.

We compare our results with three specialized methods for this task (Staal $\mathrm{SAN}^{+} 04$, Niemeijer $\left[\mathrm{NSvG}^{+} 04\right.$ and Zana [ZK01]). We trained a KA operator with a Gaussian kernel with $\gamma=0.01$ and 200,000 examples (as determined in Section 6.1). We used the NILC-DT and NILC-KA operators from Section 6.3. The windows selected by NILC-DT are shown in Figure 7.7. The results are shown in Table 7.2.

All learning based methods scored fairly well. KA trained with a $13 \times 13$ 


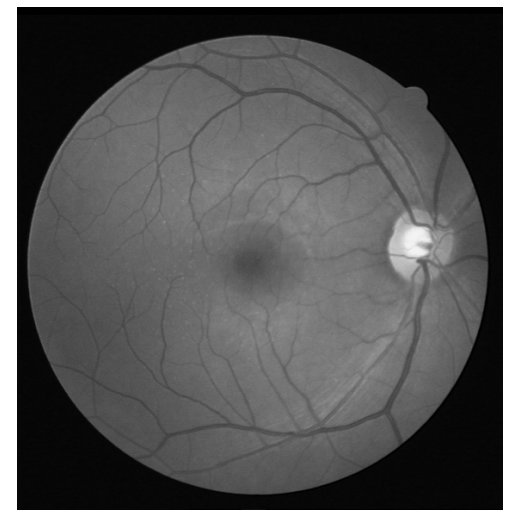

(a) Input.

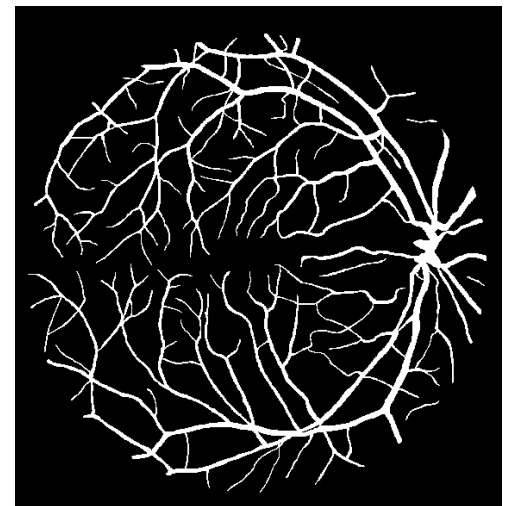

(b) Expected output

Figure 7.6: Input-output pair for the vessel segmentation task.
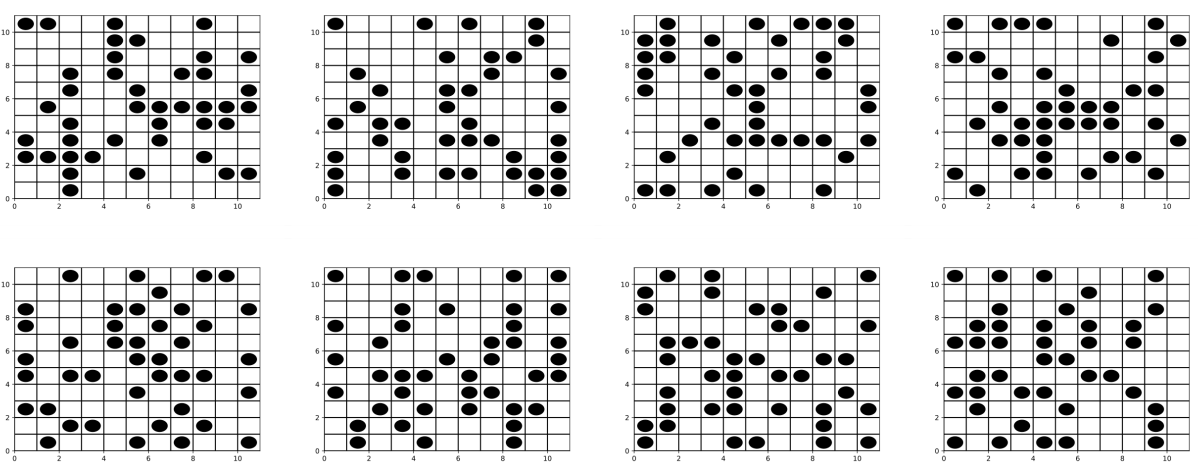

Figure 7.7: Windows selected by NILC-DT. There is not a clear pattern in the pixel distribution. Black points are used as features in training.

\begin{tabular}{|l|c|}
\hline Method & Accuracy (\%) \\
\hline KA11 & 94.34 \\
KA13 & $\mathbf{9 4 . 4}$ \\
NILC-DT & 94.21 \\
NILC-KA & 94.25 \\
\hline \hline Staal & 94.42 \\
Niemeijer & 94.16 \\
Zana & 93.77 \\
\hline
\end{tabular}

Table 7.2: Results for the DRIVE dataset. 
window had the best results, although all other operators were less than 0.2 points behind. As seen in the Experiments chapter, combining KA operators does not increase performance. The impressive results obtained by NILCDT in the validation set were also present in the test set. This shows that NILC-DT did not overfit.

A comparison of the result of all methods is shown in Figure 7.8. 11 The difference in accuracy between the learned operators and the heuristic methods seems to be related to the amount of noise present in the outputs. All tested methods correctly identified the general structure of the vessels but failed to segment thin vessels. Between the learned operators, NILC-DT produced the noisiest results, while KA13 had the cleanest images. Zana produced the cleanest image of all, but missed many thin vessels. Niemeijer had this same issue, but to a lesser extent. Finally, Stall produced fairly connected output and without much noise. Visually, producing a connected output seems more important than getting an exact copy of the expected output. Integrating connectivity in the learning process may help to reduce the false positives in the output images.

\footnotetext{
${ }^{1}$ The results of Staal, Zana and Niemeijer were obtained from http://www.isi.uu.nl/ Research/Databases/DRIVE/browser.php
} 


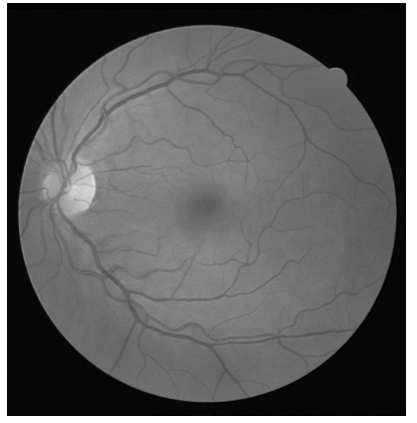

(a) Input

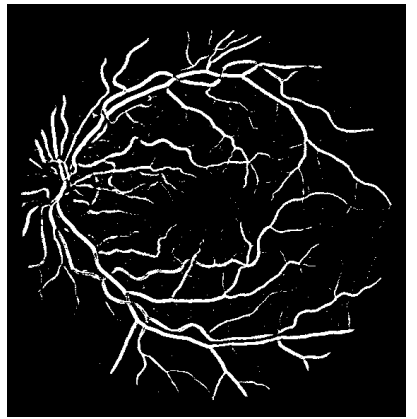

(d) KA13

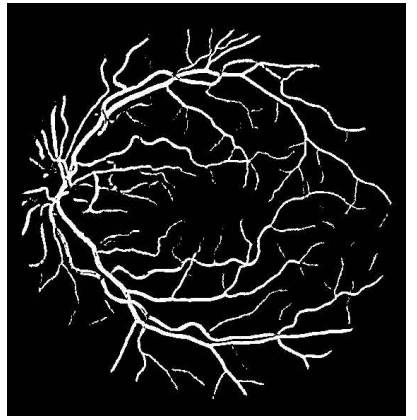

(g) Staal

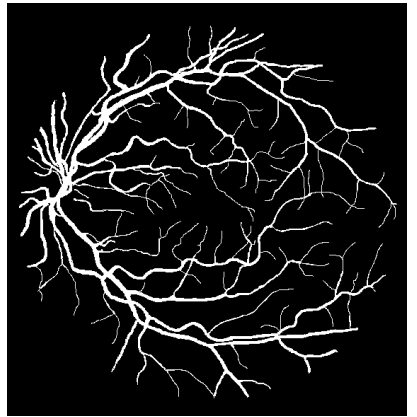

(b) Expected output

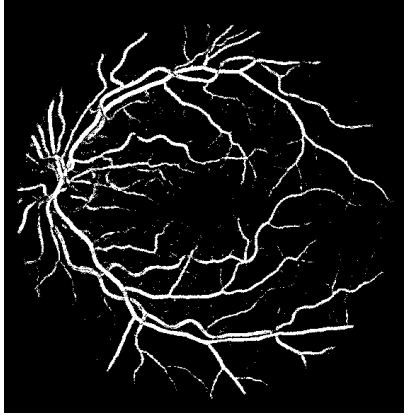

(e) NILC-DT

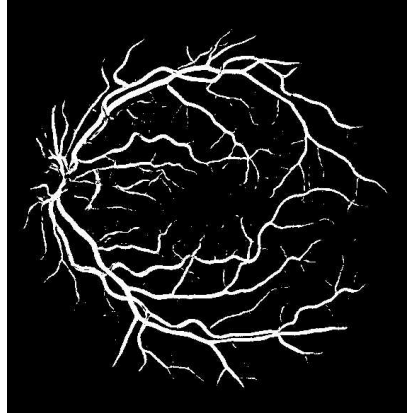

(h) Niemeijer

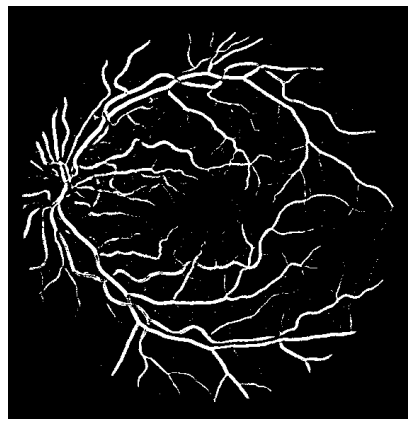

(c) KA11

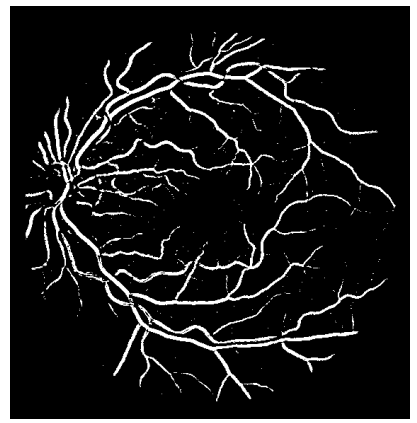

(f) NILC-KA

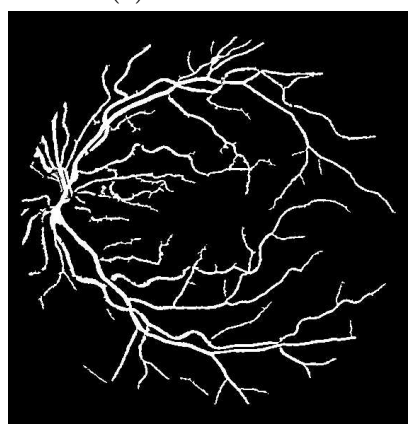

(i) Zana

Figure 7.8: Result images for the DRIVE dataset. 


\section{Chapter 8}

\section{Conclusion}

In this chapter we review the key contributions of this thesis and present some possibilities to further improve both the methods presented and $W$ operator learning as a whole.

The central objective of this work was to bring $W$-operator learning closer to "just works" status, with four concrete objectives: (i) improve training using gray-level inputs and large windows; (ii) reduce parameter determination; (iii) create a library with the implemented methods; and (iv) apply $W$-operator learning to real data. The methods presented in this thesis fulfill objectives (i), (ii) and (iv), while TRIOSlib fulfills (iii). Operators trained with KA and NILC have simpler parameter determination than previous methods and have obtained good results in both binary and gray-level inputs. These methods were also evaluated in public datasets and compared to other methods from the literature. In special, learned operators achieved state-of-the-art results in the Staff Removal problem.

The main theoretical contribution of this work is the use of continuous optimization methods to train $W$-operators. Instead of being represented by discrete structures such as lattices, both operator parameters and image data are represented using points in $\mathbb{R}^{M}$. This change impacts how operators are represented and what operations can be done using image data. Convex optimization methods are particularly suited for $W$-operator learning, as they always produce a global minimum.

In KA both the data representation and the classifier's parameters are continuous. First, KA transforms the feature space from a discrete set of pixel values to a point in $\mathbb{R}^{m}$. Then is does classification using SVMs, which rely on the concept of margin to achieve good generalization. Even though operations do not act on image data, visual interpretation of the results is achieved by the choice of kernel used.

In the case of NILC, the second level operator is a linear model trained using a continuous loss function. The analysis of the subgradients of the loss allows us to do feature selection and error minimization at the same 
time. The weight of each operator represents its relative importance in the combination and the final result resembles a weighted sum of the result images from all first-level operators.

As shown in the experiments, these contributions successfully tackle the three main challenges in the current state-of-the-art. First, they produce operators that have good performance even when trained using large windows. Second, NILC greatly reduces the number of parameters for training two-level operators while defining a clear optimization goal for two-level operator learning. Third, both methods were successfully applied to the design of gray-level operators.

This thesis also had some practical contributions worth of notice. First, it employed $W$-operator learning to datasets containing a much larger number of images than before. Both staffs and DRIVE are used by many other researchers and we compared our results with methods from the literature. We hope that future research will use these datasets and other public ones to push the boundaries on what problems can be solved using $W$-operator learning.

The second practical contribution is the development of TRIOSlib, a Python library containing all presented methods and many tools to ease the implementation of $W$-operator learning techniques. This allows other researchers to reproduce our results and to build on top of our work, as some have already done. TRIOSlib in itself is an evolution of PAClib, a C library for $W$-operator learning built in the early 2000s.

This work has only started to introduce the use of continuous optimization techniques in the context of $W$-operator learning. We list below some opportunities for future work using these methods.

1. Learning from huge datasets: the size of datasets has increased exponentially in the last decade. The DRIVE dataset, created in 2004, contains a total of 40 images. The staffs dataset, assembled in 2013, contains 4000 images. Stochastic optimization methods have gained importance in the last decade due to their successful application in this huge data scenario. Also, many stochastic methods have efficient parallel implementations using GPUs that offer dramatic speedups when compared to sequential implementations. The application of stochastic optimization in $W$-operator learning could help exploiting the information contained in a much bigger number of images.

2. Hyperparameter determination: in the context of $W$-operator learning, hyperparameter determination is unpractical due to the need of experience and deep knowledge about the models used. The behavior of many types of hyperparameters was already studied for some continuous optimization methods and this could be exploited to reduce user intervention during training. 
3. Image representations: both KA and NILC use a continuous representation for image data instead of the raw pixels, which have a discrete structure. Many tasks studied in Computer Vision, such as texture recognition and keypoint description, also compute different representations for image data. Can we explore these other representations to build $W$-operators? If using this representations is beneficial, can we determine automatically which representation is more suited to each task?

A final point of future work is the use of $W$-operator learning to solve higher-level tasks where success is measured using a domain specific measure. For instance, Optical Character Recognition is successful even when a certain level of noise is present. In these higher-level tasks pixel error may not correlate directly with the measure of interest and mistakes may not all have the same weight. It might be more advantageous to miss more pixels as long as the important one are correctly classified. The behavior of learned $W$-operators has not been studied under these contexts and applying $W$ operators to solve more complicated problems will surely motivate advances in the field. 


\section{Bibliography}

[AK98] Edoardo Amaldi e Viggo Kann. On the approximability of minimizing nonzero variables or unsatisfied relations in linear systems. Theoretical Computer Science, 209(1-2):237-260, 1998.

[AM] Yaser S Abu-Mostafa. Learning from data, volume 4.

[BB91] G. J. F. Banon e J. Barrera. Minimal Representations for Translation-Invariant Set Mappings by Mathematical Morphology. SIAM J. Applied Mathematics, 51(6):1782-1798, December 1991.

[Bru02] Marcel Brun. Projetos de operadores morfológicos multi-escala por otimização estatística. Tese de Doutorado, Instituto de Matemática e Estatística - USP, 2002.

[BTHH00] J. Barrera, R. Terada, R. Jr. Hirata e N.S.T. Hirata. Automatic programming of morphological machines by pac learning. $A n-$ nales Societatis Mathematicae Polonae. Series 4: Fundamenta Informaticae, Vol. 41, Nr 1,2:229-258, 2000.

[CCZTR16] Vicente Bosch Campos, Jorge Calvo-Zaragoza, Alejandro H Toselli e Enrique Vidal Ruiz. Sheet music statistical layout analysis. Em Frontiers in Handwriting Recognition (ICFHR), 2016 15th International Conference on, páginas 313-318. IEEE, 2016.

[CT12] Thomas M Cover e Joy A Thomas. Elements of information theory. John Wiley \& Sons, 2012.

[DDPF08] Christoph Dalitz, Michael Droettboom, Bastian Pranzas e Ichiro Fujinaga. A comparative study of staff removal algorithms. IEEE Trans. Pattern Anal. Mach. Intell., 30(5):753766, 2008.

[DH15] M.M. Dornelles e N.S.T. Hirata. Selection of windows for woperator combination from entropy based ranking. Em 28th 
Conference on Graphics, Patterns and Images (SIBGRAPI), páginas 64-71, Aug 2015.

[Dou92] Edward R. Dougherty. Optimal mean-square n-observation digital morphological filters: I. optimal binary filters. CVGIP: Image Understanding, 55(1):36 - 54, 1992.

[dSdS10] Carlos da Silva dos Santos. Construção de atributos binários baseada em análise de interações. Tese de Doutorado, Instituto de Matemática e Estatística - USP, 2010.

[FDGL12] Alicia Fornés, Anjan Dutta, Albert Gordo e Josep Lladós. Cvcmuscima: a ground truth of handwritten music score images for writer identification and staff removal. International Journal on Document Analysis and Recognition (IJDAR), 15(3):243-251, 2012.

[Fle04] François Fleuret. Fast binary feature selection with conditional mutual information. Journal of Machine Learning Research, 5(Nov):1531-1555, 2004.

$\left[\mathrm{FVD}^{+} 13\right]$ Alicia Fornes, Muriel Visani, Anjan Dutta, Van Cuong Kieu e Nicholas Journet. The ICDAR/GREC 2013 Music Scores Competition on Staff Removal. Em Proceedings of the Tenth IAPR International Workshop on Graphics Recognition (GREC 2013), páginas 129-132, États-Unis, Setembro 2013.

[FYR11] Rémi Flamary, Florian Yger e Alain Rakotomamonjy. Selecting from an infinite set of features in svm. Em European Symposium on Artificial Neural Networks, 2011.

[Gí4] Thierry Géraud. A morphological method for music score staff removal. Em Proceedings of the 21st International Conference on Image Processing (ICIP), páginas 2599-2603, Paris, France, 2014.

[GE03] Isabelle Guyon e André Elisseeff. An introduction to variable and feature selection. The Journal of Machine Learning Research, 3:1157-1182, 2003.

[GGNZ06] Isabelle Guyon, Steve Gunn, Masoud Nikravesh e Lotfi A. Zadeh. Feature Extraction: Foundations and Applications (Studies in Fuzziness and Soft Computing). Springer-Verlag New York, Inc., Secaucus, NJ, USA, 2006.

[Grü07] Peter D Grünwald. The minimum description length principle. MIT press, 2007. 
[GW06] Rafael C. Gonzalez e Richard E. Woods. Digital Image Processing (3rd Edition). Prentice-Hall, Inc., Upper Saddle River, NJ, USA, 2006.

[HBBD06] R. Hirata Jr., M. Brun, J. Barrera e E. R. Dougherty. Aperture filters: theory, application, and multiresolution analysis. Em S. Marshall e G. L. Sicuranza, editors, Advances in Nonlinear Signal and Image Processing, volume 6 of EURASIP Book Series on Signal Processing and Communications, páginas 15-48. Hindawi, 2006.

[Hei94] H. J. A. M. Heijmans. Morphological Image Operators. Academic Press, Boston, 1994.

[HFL16] Nina ST Hirata, Mariela A Fernandez e Rubens M Lopes. Plankton image classification based on multiple segmentations. Em Computer Vision for Analysis of Underwater Imagery (CVAUI), 2016 ICPR 2nd Workshop on, páginas 55-60. IEEE, 2016.

[Hir09] Nina S. T. Hirata. Multilevel training of binary morphological operators. IEEE Transactions on Pattern Analysis and Machine Intelligence, 31(4):707-720, April 2009.

[HRB07] N. S. T. Hirata, R. Hirata Jr. e J. Barrera. Basis computation algorithms. Em Mathematical Morphhology and its Applications to Signal and Image Processing (Proceedings of the 8th International Symposium on Mathematical Morphology), páginas 15-26, 2007.

[HTF09] Trevor Hastie, Robert Tibshirani e Jerome Friedman. The Elements of Statistical Learning: Data Mining, Inference, and Prediction. Springer, second edição, 2009.

[KR92] Kenji Kira e Larry A. Rendell. The feature selection problem: traditional methods and a new algorithm. Em Proceedings of the tenth national conference on Artificial intelligence, AAAI'92, páginas 129-134. AAAI Press, 1992.

[KRSRP96] Igor Kononenko, Marko Robnik-Sikonja, Marko Robnik e Uros Pompe. Relieff for estimation and discretization of attributes in classification, regression, and ilp problems, 1996.

[Kun04] Ludmila I. Kuncheva. Combining Pattern Classifiers: Methods and Algorithms. Wiley, 2004. 
[MCB06] Jr Martins, David C., Jr Cesar, Roberto M. e Junior Barrera. W-operator window design by minimization of mean conditional entropy. Pattern Analysis and Applications, 9(2-3):139$153,2006$.

[McG54] William J McGill. Multivariate information transmission. Psychometrika, 19(2):97-116, 1954.

[MHH14a] Igor Montagner, Roberto Hirata Jr. e Nina S. T. Hirata. Learning to remove staff lines from music score images. Em IEEE International Conference on Image Processing (ICIP), páginas 2614 - 2618, Paris, France, Outubro 2014.

[MHH14b] Igor Montagner, Roberto Hirata Jr. e Nina S T Hirata. A machine learning based method for staff removal. Em 22nd International Conference on Pattern Recognition (ICPR), páginas $3162-3167,2014$.

[MHH16] Igor S Montagner, Nina ST Hirata e Roberto Hirata. Staff removal using image operator learning. Pattern Recognition, 2016.

[MSHC] Igor S. Montagner, Nina S. T. Hirata, Roberto Hirata Jr. e Stéphane Canu. NILC: a two level learning algorithm with operator selection. Em To appear at IEEE International Conference on Image Processing 2016 (ICIP 2016).

$\left[\mathrm{NSvG}^{+} 04\right]$ M. Niemeijer, J.J. Staal, B. van Ginneken, M. Loog e M.D. Abramoff. Comparative study of retinal vessel segmentation methods on a new publicly available database. Em J. Michael Fitzpatrick e M. Sonka, editors, SPIE Medical Imaging, volume 5370, páginas 648-656. SPIE, SPIE, 2004.

$\left[\mathrm{RFP}^{+} 12\right]$ Ana Rebelo, Ichiro Fujinaga, Filipe Paszkiewicz, Andre RS Marcal, Carlos Guedes e Jaime S Cardoso. Optical music recognition: state-of-the-art and open issues. International Journal of Multimedia Information Retrieval, 1(3):173-190, 2012.

[RR09] Ali Rahimi e Benjamin Recht. Weighted sums of random kitchen sinks: Replacing minimization with randomization in learning. Em D. Koller, D. Schuurmans, Y. Bengio e L. Bottou, editors, Advances in Neural Information Processing Systems 21, páginas 1313-1320. Curran Associates, Inc., 2009.

$\left[\mathrm{SAN}^{+} 04\right]$ J.J. Staal, M.D. Abramoff, M. Niemeijer, M.A. Viergever e B. van Ginneken. Ridge based vessel segmentation in color images of the retina. IEEE Transactions on Medical Imaging, 23(4):501-509, 2004. 
[SHH10] Carlos S. Santos, Nina S.T. Hirata e Roberto Hirata Jr. An information theory framework for two-stage binary image operator design. Pattern Recognition Letters, 31(4):297-306, 2010.

[Soi03] P. Soille. Morphological Image Analysis. Springer-Verlag, Berlin, 2nd edição, 2003.

[SS01] Bernhard Scholkopf e Alexander J. Smola. Learning with Kernels: Support Vector Machines, Regularization, Optimization, and Beyond. MIT Press, Cambridge, MA, USA, 2001.

[tri16] Training image operators from samples, http://trioslib.sf.net/, Agosto 2016.

[Vap13] Vladimir Vapnik. The nature of statistical learning theory. Springer Science \& Business Media, 2013.

[VKFJ13] M. Visaniy, V.C. Kieu, A. Fornes e N. Journet. ICDAR 2013 music scores competition: Staff removal. Em 12th International Conference on Document Analysis and Recognition (ICDAR), páginas 1407-1411, 2013.

[WN99] Stephen Wright e Jorge Nocedal. Numerical optimization. Springer Science, 35:67-68, 1999.

[Wol92] D. H. Wolpert. Stacked generalization. Neural Networks, 5:241259, 1992.

[WS01] Christopher K. I. Williams e Matthias Seeger. Using the Nyström method to speed up kernel machines. Em T. K. Leen, T. G. Dietterich e V. Tresp, editors, Advances in Neural Information Processing Systems 13, páginas 682-688. MIT Press, 2001.

[YLM $\left.{ }^{+} 12\right]$ Tianbao Yang, Yu-Feng Li, Mehrdad Mahdavi, Rong Jin e ZhiHua Zhou. Nyström method vs random fourier features: A theoretical and empirical comparison. Em Advances in neural information processing systems, páginas 476-484, 2012.

[ZH05] Hui Zou e Trevor Hastie. Regularization and variable selection via the elastic net. Journal of the Royal Statistical Society: Series B (Statistical Methodology), 67(2):301-320, 2005.

[ZK01] Frederic Zana e Jean-Claude Klein. Segmentation of vessel-like patterns using mathematical morphology and curvature evaluation. IEEE Transactions on Image Processing, 10(7):10101019, 2001. 